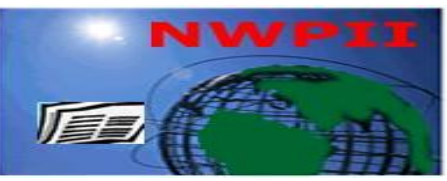

American Journal of Biomedical Sciences

ISSN: 1937-9080

nwpii.com/ajbms

\title{
The Molecular Heterogeneity of Natural Cordyceps sinensis with Multiple Ophiocordyceps sinensis Fungi Challenges the Anamorph-Teleomorph Connection Hypotheses
}

\author{
Yu-Ling Li ${ }^{1}$, Yi-Sang $\mathrm{Yao}^{2}$, Wei-Dong $\mathrm{Xie}^{2}$, Jia-Shi $\mathrm{Zhu}^{2,3, *}$ \\ ${ }^{1}$ Qinghai University, Institute of Grassland Research, Qinghai Academy of Animal Husbandry and Veterinary Sciences, \\ Xining, Qinghai 810016 China. \\ ${ }^{2}$ Division of Life Sciences and Health, Tsinghua University Graduate School at Shenzhen, Guangdong 518055, China. \\ ${ }^{3}$ Department of Applied Biology and Chemistry Technology, The Hong Kong Polytechnic University, Hong Kong. \\ *Corresponding Author \\ Jia-Shi Zhu \\ Division of Life Sciences and Health \\ Tsinghua University Graduate School at Shenzhen \\ Guangdong 518055, China \\ Department of Applied Biology and Chemistry Technology \\ The Hong Kong Polytechnic University \\ Hong Kong \\ Tel: +18587053789 \\ Fax: +18587775435 \\ E-mail: zhujosh@gmail.com
}

Received: 21 December 2015; | Revised: 27 March 2016; | Accepted: 25 April 2016

\begin{abstract}
Natural Cordyceps sinensis is a traditional Chinese medicine with a long history of use as a folk medicine in China. However, whether Ophiocordyceps sinensis is a single fungus or a collective name for multiple fungi remains controversial, as does the anamorph-teleomorph connection of $O$. sinensis. Although Hirsutella sinensis has been widely considered the sole anamorph of $O$. sinensis, direct evidence supporting this hypothesis is lacking. This paper reviews the molecular heterogeneity findings of $C$. sinensis studies and the issues with the hypotheses of the anamorph-teleomorph connection of $O$. sinensis. Molecular analyses have revealed the coexistence of multiple fungi and multiple genotypes of $O$. sinensis in natural $C$. sinensis. Mutant sequences from at least $11 \mathrm{O}$. sinensis genotypes are registered in GenBank and represent individual fungi distinct from the genome sequence of $H$. sinensis, which is inconsistent with the "sole anamorph" and "ITS pseudogenes" hypotheses. Together, the multicellular $C$. sinensis ascospores with mono-/bi-/trinucleate structures in each ascospore and the detection of at least $2 O$. sinensis genotypes in the culture of heterokaryotic single-ascospore isolates challenge the study conclusions based on microcycle conidiation of C. sinensis ascospores. During $C$. sinensis maturation, the fungi that grow differentially in the caterpillar body and stroma of $C$. sinensis undergo asynchronous, dynamic alterations, supporting the integrated microecosystem hypothesis for natural $C$. sinensis proposed by Prof. Zongqi Liang.
\end{abstract}


Keywords: Molecular systematics of Cordyceps sinensis; mutant genotypes of Ophiocordyceps sinensis; insect-fungi complex; anamorph-teleomorph connection; binucleate; trinucleate; multicellular heterokaryons.

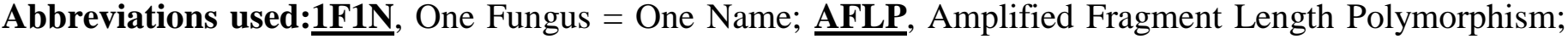
CAPS, Cleaved Amplified Polymorphic Sequence; DAF, DNA Amplified Fingerprints; gb, GenBank; $\underline{\text { ICTF}}$, International Commission on the Taxonomy of Fungi; IMA, International Mycology Association;

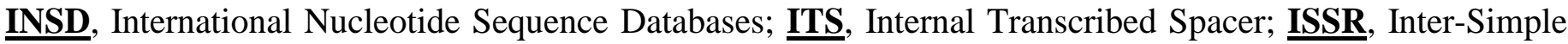
Sequence Repeat; MALDI-TOF, Matrix-Assisted Laser Desorption/Ionization-Time Of Flight; MS, Mass Spectrum or Mass Spectrometry or Mass Spectrogram or Mass Spectrograph; nrDNA, nuclear ribosomal DNA; OTUs, Operational Taxonomic Units; PCR, Polymerase Chain Reaction; qPCR, quantitative Polymerase Chain Reaction; RAPD, Random Amplified Polymorphic DNA; RFLP, Restriction Fragment Length Polymorphism; SCAR, Sequence Characterized Amplified Regions; SNP, Single Nucleotide Polymorphism; SSCP, Single-Strand Conformation Polymorphism; SSR, Simple Sequence Repeat; $\underline{\text { TCM, }}$ Traditional Chinese Medicine; UPGMA, Unweighted Pair Group Method with Arithmetic Mean.

Natural Cordyceps sinensis is the most expensive substance in traditional Chinese medicine (TCM), with a rich history of traditional use in China for "Yin-Yang" double invigoration, health maintenance, disease amelioration, postdisease recovery, and anti-aging therapy [1-2]. (Section 1, below, addresses the controversy surrounding the indiscriminate use of the Latin names for the natural wild product and the fungi.) Morphological and TCM descriptions of this medicinal substance in ancient TCM books as a unique therapeutic entity can be traced back to the $15^{\text {th }}$ century [3]. Modern pharmacological examinations have validated the therapeutic profile of wild $C$. sinensis and its mycelial fermentation products [4-6]. We also demonstrated pharmacologically the lifespanextending properties of a fermentation product of a $C$. sinensis isolate, Paecilomyces hepiali Cs-4, and its ability to reverse aging-induced changes in genome-wide gene expression patterns [7]. Natural $C$. sinensis grows only in alpine areas above 3,000-3,500 $\mathrm{m}$ on the Qinghai-Tibetan Plateau and has a complex life cycle [1,4,7-9]. The development-maturation stages of natural $C$. sinensis greatly impact its therapeutic efficacy and potency and are used as a market standard for grading the quality of natural $C$. sinensis [10-13].

The anamorph-teleomorph connection of Ophiocordyceps sinensis has been the subject of a decades-long academic debate. Based on the collection of indirect evidence obtained using several techniques, some mycologists agree that
$H$. sinensis is the sole anamorph of $O$. sinensis [14-15]. However, to date, no direct evidence strictly and fully satisfying Koch's Postulates (association, isolation/purification, re-inoculation, and re-isolation) has been documented. The artificial production of sexual fruiting bodies and ascospores from postulated anamorphic fungal strains previously isolated from natural $C$. sinensis specimens, purified and cultured to inoculate the host (larvae of the family Hepialidae), has repeatedly proven unsuccessful [4-22]. Guo et al. [15] and Xiao et al. [20] reviewed indirect evidence for the "sole $H$. sinensis anamorph" hypothesis using multiple technologies, including the isolation and morphological examination of fungal strains, the microcycle conidiation of ascospores, and molecular systematics studies. Unfortunately, the majority of the fungal species in the natural world cannot be cultured, making it difficult to conduct studies using traditional techniques of fungal isolation, purification and morphological identification and microcycle conidiation [18,2326]. Culture-independent molecular systematics studies of natural $C$. sinensis specimens have matured over the last 18 years and have generated indirect evidence for the assessment of the anamorph-teleomorph connection of $O$. sinensis. This review describes molecular biology studies of natural $C$. sinensis specimens and related scientific issues. 
1. Indiscriminate use of the Latin name $C$. sinensis or $\boldsymbol{O}$. sinensis for the wild product and the fungi

We begin this review with a discussion of the controversy concerning the Latin name Cordyceps sinensis (Berkeley) Saccardo, which has been used indiscriminately for both the wild product, which contains multiple fungi and the dead body of a larva of the family Hepialidae, and for the teleomorph and holomorph of $C$. sinensis fungus/fungi [3,14-16,27-30]. The indiscriminate use of the single Latin name can be traced back to the original literature published in 1843 and 1857, when British mycologist Miles Joseph Berkeley examined the fungal species in natural $C$. sinensis [3,30-34]. The fungus/fungi were re-named to the synonym Ophiocordyceps sinensis (Berkeley) Sung et al. [35], while the name of the wild product remains unchanged. Because of the indiscriminate practice that has created confusion among hundreds of publications, Ren et al. [36] proposed the use of "Ophiocordyceps \& Hepialidae" to reflect the nature of the insect-fungi complex of the wild product. However, that proposal has not been generally accepted because the fungi currently under the name of $O$. sinensis may or may not belong to the genus Ophiocordyceps (see below for discussion). Zhang et al. [37], on the other hand, proposed the use of "Chinese cordyceps" for the wild product and $O$. sinensis for the fungus/fungi. However, the use of the non-Latin name Chinese cordyceps for the wild product never reached general consensus and was unfortunately rejected by taxonomists in TCM botany because of the general practice and governmental regulation that every TCM product must be given an exclusive Latin name. Due to this awkward situation, Lo et al. [4], Zhao et al. [38] and many other papers simply used the Chinese alphabetic "Dong Chong Xia Cao 冬虫 夏草” for the wild product, and Zhou et al. [6] used its abbreviation "DCXC". In this review, we temporarily refer to the fungi as "Ophiocordyceps sinensis" and continue the customary use of the name Cordyceps sinensis to refer to the wild product, although this practice will likely be replaced by the discriminate use of unique Latin names.

In addition to the indiscriminate use of Latin names for the wild product and the teleomorph and holomorph of the fungus/fungi, the use of the teleomorphic name $O$. sinensis to replace the anamorphic name $H$. sinensis, the postulated anamorph of $O$. sinensis, has been proposed by Zhang et al. [28] following the Amsterdam Declaration (International Mycology Association, or IMA) of "One Fungus = One Name (1F1N)" [39-41]. This proposal, however, can only be accepted scientifically if $O$. sinensis is indeed "One Fungus" and if $H$. sinensis is truly the sole anamorph of $O$. sinensis.

\section{Is $O$. sinensis the Latin name for a single fungal species?}

As a prerequisite for implementing the $1 \mathrm{~F} 1 \mathrm{~N}$ declaration [39-41] in natural $C$. sinensis research [28], O. sinensis must represent a single fungal species. For the $O$. sinensis fungus/fungi, however, the following 3 hypotheses from the literature require scientific validation:

2.1 $O$. sinensis is a single fungus, and $H$. sinensis is the sole anamorph of $O$. sinensis [4,14-15,28]

If this hypothesis is accurate, the anamorphic name $H$. sinensis could be replaced with the teleomorphic name $O$. sinensis. In a comparison of the sequence of $H$. sinensis that had been isolated and purified using currently available techniques with the database sequences of $O$. sinensis, Zhang et al. [29] stated that all $O$. sinensis sequences registered in the International Nucleotide Sequence Databases (INSD; GenBank is one member of the INSD), except for Group A (H. sinensis), should be "treated as incorrect sequences" under the hypothetical assumption that $H$. sinensis is the sole anamorph of $O$. sinensis, because these sequences were "reported from natural Chinese cordyceps samples rather than from isolated fungal cultures" (more discussion below). Despite the controversies (see 2.2 and 2.3 below), many scientific publications have followed this hypothesis. 
2.2 $O$. sinensis is the collective name for multiple fungi $[6,10-13,15,17-18,25,27,42.44-$ 51]

Jiang and Yao [17] summarized the isolation of 22 fungal species spanning 13 genera from natural $C$. sinensis specimens and reiterated the criteria for adequately confirming the correct anamorph of $O$. sinensis. According to these criteria [17-18], no report to date concerning any of the 22 fungal species has strictly and fully satisfied Koch's Postulates, leading to no direct evidence to prove or disprove any of the fungal species as the true anamorph of $O$. sinensis. Dong et al. [10] stated that "more than 20 anamorphic fungi were isolated from natural Chinese cordyceps and reported to be connected with the teleomorph of $O$. sinensis". Barseghyan et al. [27] confirmed that both $H$. sinensis and Tolypocladium sinense "were identified as the anamorphs of Ophiocordyceps sinensis". It is worth noting that Yang [21] previously speculated about hyperparasitism for $O$. sinensis and that Bushley et al. [52] reported the fluorescent-stained binucleate structure of hyphae and the mono-/bi-/trinucleate structures of ascospores of $C$. sinensis (Figure 3 of [52]). Xia et al. [48] used real-time qPCR and cloningsequencing to profile the microbiota of natural $C$. sinensis samples collected from Nagqu in Tibet. They reported ITS sequences of 97 fungal clones and identified multiple fungi ( $\mathrm{gb}$ KJ734995 KJ735091), including dominant fungal species or Operational Taxonomic Units (OTUs) from the genera Geomyces, Phoma, and Trichocladium in the caterpillar body of $C$. sinensis and dominant species or OTUs from the genera Geomyces and Cladosporium in the stroma, in addition to the $16 \mathrm{~S}$ sequences of 97 bacterial clones ( $\mathrm{gb}$ KJ717845 - KJ717941). However, they did not detect $H$. sinensis ITS sequences from either the caterpillar body or the stroma of $C$. sinensis [48]. Similar findings were reported for both culturedependent and culture-independent techniques by Zhang et al. [25,51], who detected different fungal dominancy from the caterpillar body and the stroma of $C$. sinensis but no $H$. sinensis in either compartment.
2.3 $O$. sinensis is the collective name for multiple genotypes of fungi with multiple, scattered transition, transversion, and/or insertion/deletion point mutations, which probably evolved from the same genetic ancestor [12,13 26,60,49-50,53-59]

A BLAST search can identify hundreds of sequences registered in NCBI GenBank under the taxid 72228 and the name $C$. sinensis or $O$. sinensis. These sequences include at least 12 mutant genotypes of $O$. sinensis and can be grouped into 3 categories $[13,50]$ : (1) 6 genotypes with multiple, scattered transition point mutations (Figures 1-3; Table 1); (2) 5 genotypes with large numbers of multiple, scattered point transversion mutations, in addition to some transition mutation bases (Figures $2 \& 4$; Tables 2-3); and (3) 3 O. sinensis sequences with multiple, scattered insertion and/or deletion mutations (ratio of Insertion/Deletion bases $v s$. Transversion/Transition mutant bases $>1.0 ; c f$. Figures $2 \& 4$, Tables 2-3).

The first 3 of the 6 transition mutant genotypes in Category 1 are GC rich ( $c f$. Figures $1 \& 2$, Table 1): Genotype \#1 (H. sinensis) is represented by AB067721 (AT=36.7\%). Genotype \#2 is a truncated form of Genotype \#1 $H$. sinensis (the 320-bp amplicon shown in the left panel of Figure 3) with a distinct maturation pattern compared with Genotype \#1 H. sinensis, shown as the 440(A)-bp amplicon in the left panel of Figure 3 [12]. Genotype \#3 is a group of sequences represented by HM595984 (AT $=36.7 \%$ ) with $94.3 \%$ and $93.0 \%$ similarity with the ITS1 and ITS2 segments of Genotype \#1 AB067721 ( $c f$. Table 1), as well as 8 additional sequences as of July 2015: FJ654148, FJ654149, JQ286748, KM197540, KJ175197, KJ175199, KJ175203, KJ175205, and KJ175206 (98\%-99\% homology among the sequences in this group Genotype \#3). Notably, the Genotype \#3 sequences FJ654148, FJ654149, and JQ286748 were incorrectly included in Group A (Genotype \#1) by Zhang S et al. [29]. 
AB0 67721

HMY595984

AB0 67744

AB0 67740

EU555436

KJ720572

KP731802

AB0 67721 HMY595984

AB0 67744

AB0 67740

EU555436

KJ720572

KP731802

AB0 67721 HM5 95984

AB067744

AB067740

EU555436

KJ720572

KP731802

AB0 67721 HMY595984

AB067744

AB067740

EU555436

KJ720572

KP731802

AB0 67721 HM1595984 AB0 67744 AB0 67740 EU555436 KJ720572 KP731802

AB0 67721 HM5 95984 AB067744 AB0 67740 EU555436

KJ720572

KP731802

AB0 67721 HMI595984 AB067744

AB0 67740 EU555436

KJ720572

KP731802
(1) T

(1)

(1)

(1)

(1)

(1)

(1)
TAGAGGAAGTAAAAGT CGTAACAAGGTCTCCGTTGGTGAACCAGCGGAGGGATCATTA.TCGAGT CACCACTCCCAAACCCCCTGCGAACACCACAGCAGT ----$---\mathrm{T}-\mathrm{TC}--$ $--\mathrm{T}--\mathrm{T}-\mathrm{T}$ $---\mathrm{T}-\mathrm{TC}--$

$\mathrm{T}-------\mathrm{A}----\mathrm{T}--\mathrm{T}-\mathrm{T}$

101

200 (101) TGCCTCGGCGGGACCGCCCCGGCGCCCCAGGGCCCGGACCAGGGCGCCCGCCGGAGGACCCCCAGACCCTCCTGTCGCAGTGGCATCTCTCAGTCAAGAA

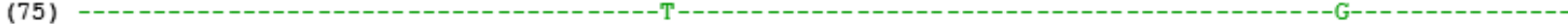
(97) ----

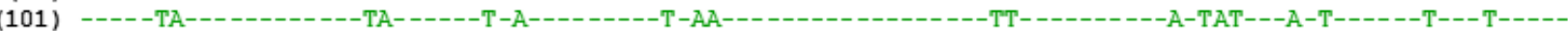
(15) ----

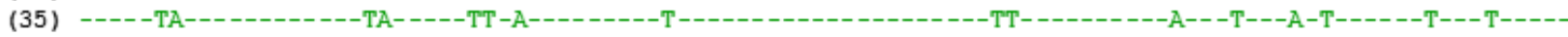

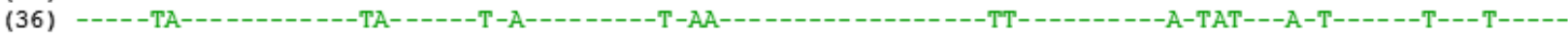

201

300

(201) GCAAGCAAATGAATCAAAACTTTCAACAAAGGATCTCTTGGTTCTGGCATCGATGAAGAACGCAGCGAAATGCGATAAGTAATGTGAATTGCAGAATTCA.

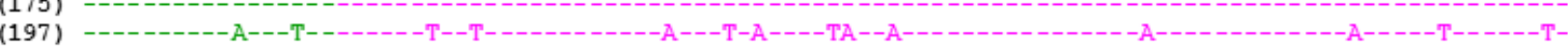

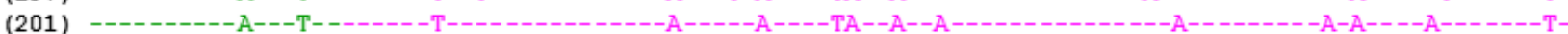

(115) ---------A---T------ -T--------------A-----A--------A-------T--TA--------------A-A----A-------T-

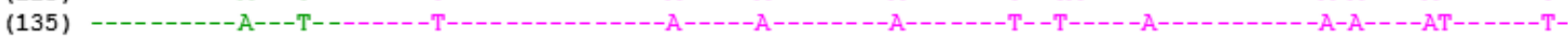

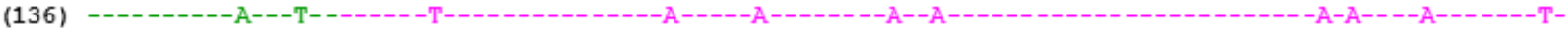

$301 \quad 400$

(301) GTGAACCATCGAATCTTTGAACGCACATTGCGCCCGCCAGCACTCTGGCGGGCATGCCTGTCCGAGCGTCATCTCAACCCTCGAGCCCCCCGCCTCGCGG

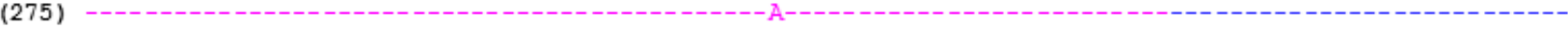

(297) --A---T-----------A--- $-\mathrm{T}-\mathrm{T}-\mathrm{C}-\mathrm{A}-------\mathrm{T}--------\mathrm{A}----------------------\mathrm{T}-\mathrm{C}--\mathrm{T}------\mathrm{T} A-----------------$

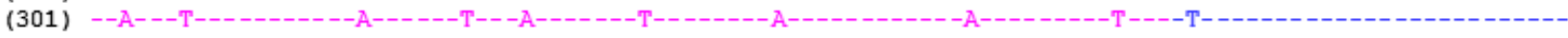

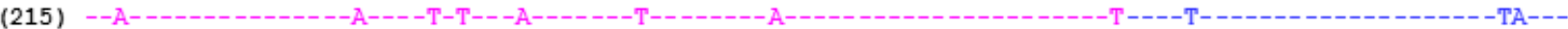

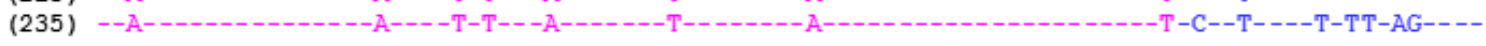

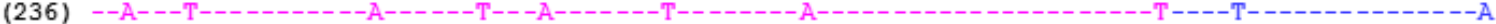

401500

(401) CGGCGGGGCCCGGCCTTGGGGGTCACGGCCCCGCGCCGCCCCCTAAACGCAGTGGCGACCCCGCCGCGGCTCCCCTGCGCAGTAGCTCGCTGAGAACCTC

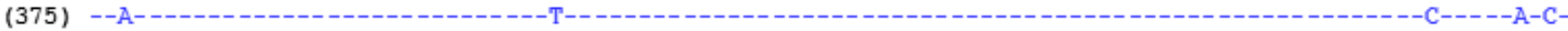

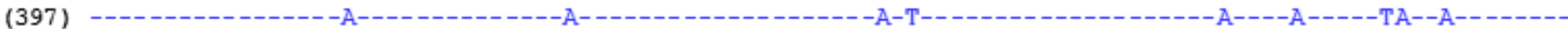

(401) --------T------ A---- T---------------T------T--

(315)

(325)

(327)

501

600

(501) GCACCGGGAGCGCGGAGGCGGT CACGCCGTGAAACCACCACACCCTCCAGTT GACCTCGGATCAGGTAGGGATACCCGCTGAACTTAAGCATATCAATAA.

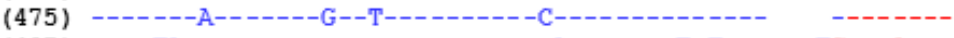

(497) --- TA----

TA

(1)

(325)

(327)

601

(601) GCGGAGGAAAAGAAACCAACAGGGATTGCCCCAGTAACGGCGAGTGAAGCGGCAACAGCTCAAATTT

(527)

(597)

(601)

(467)

(325)

(327)

Figure 1 ITS sequence alignment of 5 groups of transition point mutants of $\boldsymbol{O}$. sinensis. AB067721 represents Genotype \#1 of GC-biased $O$. sinensis fungi (including H. sinensis). HM595984 represents Genotype \#3, a GC-biased mutant genotype. AB067744 (Genotype \#4), AB067740 (Genotype \#5), and EU555436 and KJ720572 (Genotype \#6) represent 3 genotypes of AT-biased $O$. sinensis fungi, whereas KP731802 represents an AT-biased genotype with $98 \%$ homology to Genotype \#5 and 97\% homology to Genotype \#6. The residues in red were annotated in GenBank as $18 \mathrm{~S}$ (5' end region) or $28 \mathrm{~S}$ ( $3^{\prime}$ ' end region) rRNA; the residues in green were annotated as ITS1; the residues in pink were annotated as 5.8S rRNA; and the residues in blue were annotated as ITS2. Hyphens indicate identical bases, and spaces denote unmatched sequence gaps.

Am. J. Biomed. Sci. 2016, 8(2), 123-159; doi: 10.5099/aj160200123 @ 2016 by NWPII. All rights reserved 
Table 1. Segmented sequence similarities of the ITS1, 5.8S and ITS2 sequences of the GC-biased (Genotype \#1 AB067721, Genotype \#3 HM595984; $c f$. Figures 1\&2) and AT-biased (Genotype \#4 AB067744; Genotype \#5 AB067740; and Genotype \#6 EU555436) genotypes of $O$. sinensis fungi.

\begin{tabular}{|c|c|c|c|c|}
\hline & ITS1 & $5.8 \mathrm{~S}$ & ITS2 & $\begin{array}{l}\text { ITS } 1-5.8 \mathrm{~S}-\mathrm{ITS} 2 \\
\text { (excluding the } 18 \mathrm{~S} \text { and } \\
\text { 28S segments) }\end{array}$ \\
\hline AB067721 vs. HM595984 & $94.3 \%$ & $99.4 \%$ & $93.0 \%$ & $95.5 \%$ \\
\hline AB067721 vs. AB067744 & $90.6 \%$ & $85.3 \%$ & $89.2 \%$ & $88.4 \%$ \\
\hline АВ067721 vs. АВ067740 & $80.5 \%$ & $86.5 \%$ & $89.2 \%$ & $85.5 \%$ \\
\hline AB067721 vs. EU555436 & $84.7 \%$ & $87.8 \%$ & $85.8 \%$ & $86.0 \%$ \\
\hline AB067721 vs. KP731802 & $82.8 \%$ & $89.1 \%$ & - & $86.5 \%$ \\
\hline HM595984 vs. AB067744 & $87.4 \%$ & $85.9 \%$ & $83.1 \%$ & $85.4 \%$ \\
\hline HM595984 vs. AB067740 & $78.6 \%$ & $87.2 \%$ & $86.0 \%$ & $84.0 \%$ \\
\hline HM595984 vs. EU555436 & $84.5 \%$ & $88.2 \%$ & $80.8 \%$ & $84.5 \%$ \\
\hline HM595984 vs. KP731802 & $81.7 \%$ & $89.7 \%$ & - & $86.3 \%$ \\
\hline АВ067744 vs. АВ067740 & $89.9 \%$ & $92.3 \%$ & $88.0 \%$ & $91.4 \%$ \\
\hline AB067744 vs. EU555436 & $94.7 \%$ & $91.5 \%$ & $84.4 \%$ & $89.6 \%$ \\
\hline АВ067744 vs. KР731802 & $91.9 \%$ & $92.9 \%$ & - & $91.9 \%$ \\
\hline AB067740 vs. EU555436 & $96.9 \%$ & $93.6 \%$ & $92.5 \%$ & $94.1 \%$ \\
\hline AB067740 vs. KP731802 & $97.9 \%$ & $97.4 \%$ & - & $97.8 \%$ \\
\hline EU555436 vs. KP731802 & $96.9 \%$ & $96.2 \%$ & - & $96.7 \%$ \\
\hline
\end{tabular}

Note: The sequence alignment analyses for the entire ITS1-5.8S-ITS2 sequences and segmented sequences were performed using Vector NTI Advance 9 (Invitrogen) based on the $O$. sinensis sequences registered in GenBank, according to the segmentation annotations released by GenBank. "_" indicates inadequate sequence data for comparison because the ITS2 segment sequence of KP731802 is insufficiently long. 


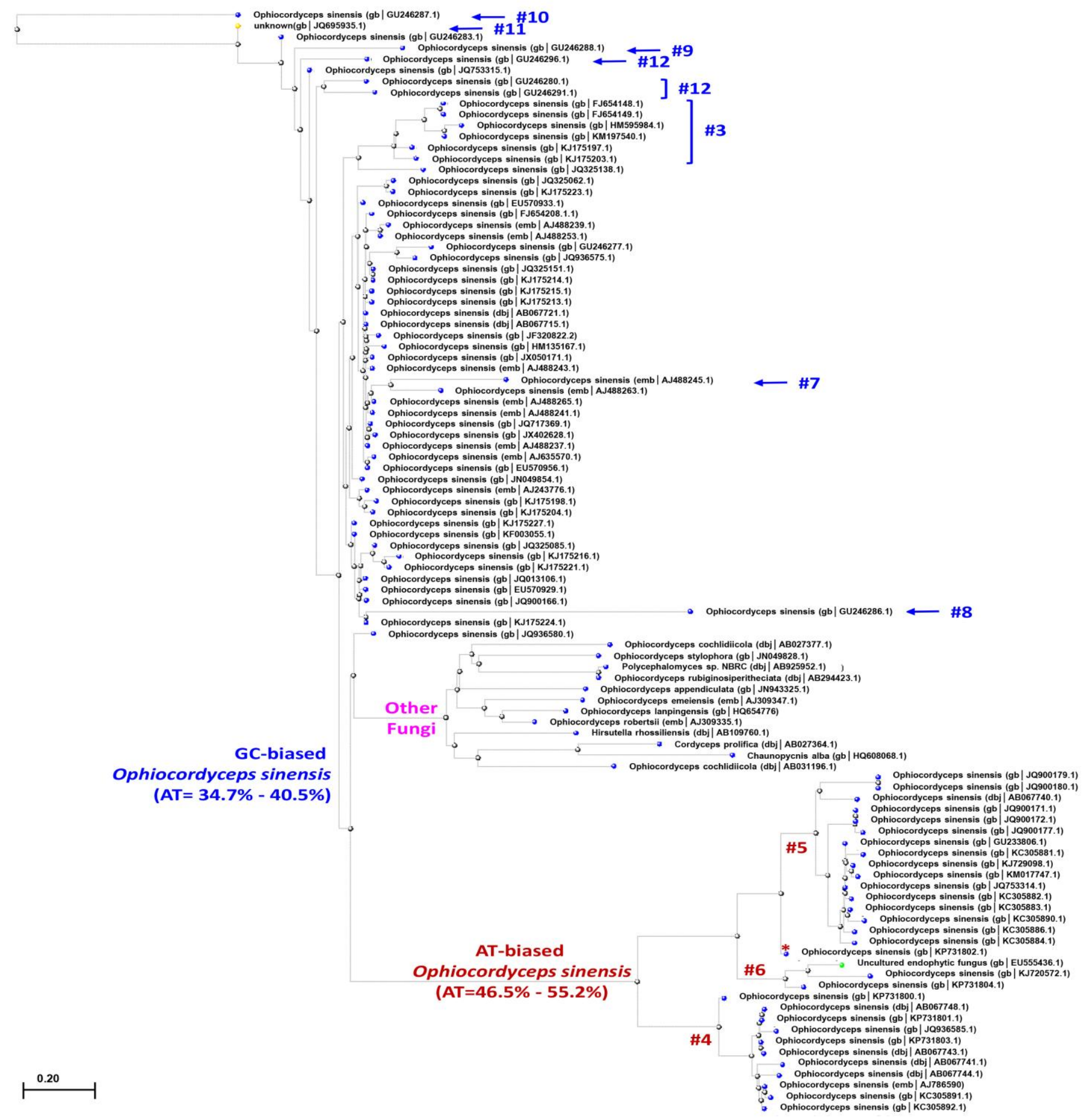

Figure 2 Phylogenetic relationship of mutant $O$. sinensis genotype fungi constructed based on their ITS sequences using the Fast Minimum Evolution (pairwise alignment) algorithm in NCBI GenBank BLAST. The $O$. sinensis sequences were grouped into 12 genotypes: Genotypes \#1-\#3 and \#7-\#12 are GC-biased genotypes; Genotypes \#4-\#6 of Category 1 are AT-biased transition point mutation genotypes. Genotypes \#1-\#6 of Category 1 are transition point mutation genotypes (see Figure 3 for Genotype \#2); Genotypes \#7-\#11 of Category 2 are transversion point mutation genotypes; and Genotype \#12 of Category 3 features a large number of insertion/deletion point mutations. *, KP731802 is highly homologous to Genotypes \#5 (98\%) and \#6 (97\%) and was placed between these 2 genotypes in this phylogenetic tree. 


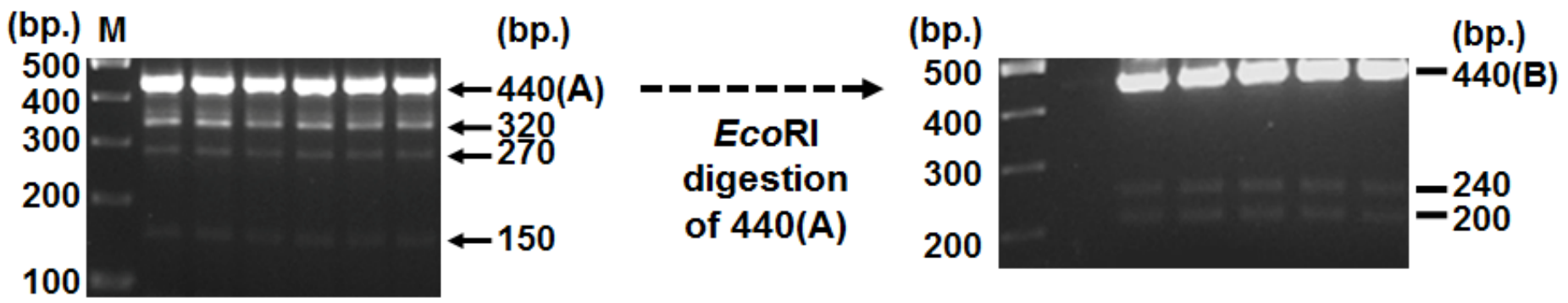

Figure 3 Agarose gel electrophoresis of the PCR amplicons (Left Panel) and the EcoRI digestion of the 440(A)bp amplicon (Right Panel) [Reproduced with permission from AJBMS (www.nwpii.com/ajbms) Am J Biomed Sci 2010; 2(3): 217-238] [26].

Left Panel: Genomic DNA templates were prepared from the premature $C$. sinensis stroma $(<1.5 \mathrm{~cm}$ in height $)$. PCR was performed using the $H$. sinensis-specific primers Hsprp1/3 and a touchdown PCR protocol. The targeted 440(A)bp amplicon shown in the left panel was recovered and sequenced, indicating $100 \%$ identity to that of Genotype \#1, the GC-biased $H$. sinensis. A 320-bp amplicon was also recovered and sequenced, revealing a truncated, short sequence (Genotype \#2) at the 5' and 3' ends and 100\% identity to GC-biased H. sinensis sequences.

Right Panel: The recovered 440(A)-bp amplicon was subjected to overnight EcoRI digestion and analyzed by agarose gel electrophoresis. A trace amount of the 440(A)-bp amplicon was digested with EcoRI (200- and 240-bp DNA moieties), representing the GC-biased transition mutant $O$. sinensis species (Genotypes \#1 \& \#3), the GC-biased transversion mutant $O$. sinensis species (Genotypes \#7-\#11), and the GC-biased deletion/insertion mutant $O$. sinensis species (Genotype \#12) ( $c f$. Figures 1 \& 4). The majority of the 440(A)-bp amplicon was not digested with EcoRI, corresponding to the 440(B)-bp DNA moiety in the right panel. The 440(B) moiety represents Genotypes \#4-\#6, the AT-biased $O$. sinensis species with a single C-to-T mutation at the 299 allele site in the AB067721 sequence, which results in the loss of the EcoRI site ( $c f$. Figure 1). The EcoRI-digestible component of the 440(A)-bp amplicon exhibited a development-maturation pattern completely different from that of the 320-bp amplicon (Genotype \#2) (Left panel) during the maturation of natural $C$. sinensis [12].

The other 3 transition mutant genotypes in Category 1 are relatively AT rich $(c f$. Figures $1 \&$ 2, Table 1): Genotype \#4, represented by AB067744 (AT=47.8\%; 103 entries in GenBank as of July 2015); Genotype \#5, represented by AB067740 (AT=51.9\%; 35 entries in GenBank as of July 2015); and Genotype \#6, represented by KJ720572 (AT=55.2\%), which shares $93 \%$ similarity with Genotype \#4 AB067744, 94\% similarity with Genotype \#5 AB067740, and <86\% similarity with the remaining $O$. sinensis genotypes, 2 other entries in this group $(98 \%$ homology): EU555436 (AT=48.7\%) and KP731804 (AT=50.4\% $\quad[12-13,26,29,30,49-$ 50,53-59,61]. Notably, Genotypes \#4 and \#5 were labeled as Groups B and C, respectively, by Stensrud et al. [53]; the Genotype \#6 sequence EU555436 was incorrectly included in Group C (Genotype \#5) by Li Yi et al. [57]. KP731802 (AT $=54.0 \%)$, one of the recently obtained ATbiased $O$. sinensis sequences in a cloning- sequencing study of the ascocarps of natural $C$. sinensis specimens, shares $98 \%$ homology with Genotype \#5 AB067740 and 97\% with Genotype \#6 EU555436 and KP731804 (cf. Figure 1, Table 1), residing between Genotypes \#5 \& \#6 in the phylogenetic tree ( $c f$. Figure 2). Further extending the ITS2 segment sequence of KP731802 towards its 28S nrDNA segment may help to determine whether KP731802 truly belongs to Genotype \#5 or \#6 or to a parental genotype of both. Mao et al. [58] reported that Genotypes \#4 and \#5 shared the same mycelial morphology as Genotype \#1 H. sinensis, but the size of the conidia was significantly greater for Genotype \#4 than for Genotype \#5. Genotypes \#5 and \#6 were predominantly detected in the stroma of natural $C$. sinensis specimens in the late maturation stages and in the ascospores, while Genotype \#4 is present in the ascocarp of natural C. sinensis $[12,54,56-57,60]$. 
Table 2 Segmented sequence similarities of the ITS1, 5.8S and ITS2 sequences of the GC-biased AB067721 (Genotype \#1 of Category 1) and transversion mutant $O$. sinensis genotypes: Genotype \#7, AJ488254; Genotype \#8, GU246286; Genotype \#9, GU246288; Genotype \#10, GU246287; Genotype \#11, JQ695935 of Category 2; and insertion/deletion point mutant $O$. sinensis Genotype \#12, GU246296 of Category 3.

\begin{tabular}{|c|c|c|c|c|}
\hline & ITS1 & $5.8 \mathrm{~S}$ & ITS2 & $\begin{array}{l}\text { ITS } 1-5.8 \mathrm{~S}-\mathrm{ITS} 2 \\
\text { (excluding the } 18 \mathrm{~S} \text { and } \\
28 \mathrm{~S} \text { segments) }\end{array}$ \\
\hline AB067721 vs. AJ488254 & $93.2 \%$ & $98.7 \%$ & $89.4 \%$ & $93.9 \%$ \\
\hline AB067721 vs. GU246286 & $86.2 \%$ & $94.8 \%$ & $87.9 \%$ & $89.6 \%$ \\
\hline AB067721 vs. GU246288 & $96.3 \%$ & $98.7 \%$ & $91.5 \%$ & $95.3 \%$ \\
\hline AB067721 vs. GU246287 & $86.2 \%$ & $92.9 \%$ & $72.4 \%$ & $83.2 \%$ \\
\hline AB067721 vs. JQ695935 & $94.3 \%$ & $100 \%$ & $55.1 \%$ & $81.6 \%$ \\
\hline AB067721 vs. GU246296 & $99.4 \%$ & $99.4 \%$ & $87.0 \%$ & $94.9 \%$ \\
\hline AJ488254 vs. GU246286 & $82.0 \%$ & $93.6 \%$ & $80.7 \%$ & $85.6 \%$ \\
\hline AJ488254 vs. GU246288 & $89.4 \%$ & $97.4 \%$ & $84.5 \%$ & $90.6 \%$ \\
\hline AJ488254 vs. GU246287 & $81.4 \%$ & $92.3 \%$ & $67.8 \%$ & $80.6 \%$ \\
\hline AJ488254 vs. JQ695935 & $88.8 \%$ & $98.7 \%$ & $59.5 \%$ & $82.8 \%$ \\
\hline AJ488254 vs. GU246296 & $92.5 \%$ & $98.1 \%$ & $77.0 \%$ & $89.5 \%$ \\
\hline GU246286 vs. GU246288 & $83.8 \%$ & $93.6 \%$ & $85.2 \%$ & $87.4 \%$ \\
\hline GU246286 vs. GU246287 & $77.5 \%$ & $87.8 \%$ & $72.0 \%$ & $78.7 \%$ \\
\hline GU246286 vs. JQ695935 & $83.8 \%$ & $94.9 \%$ & $56.3 \%$ & $77.5 \%$ \\
\hline GU246286 vs. GU246296 & $86.9 \%$ & $94.3 \%$ & $82.3 \%$ & $87.6 \%$ \\
\hline GU246288 vs. GU246287 & $84.4 \%$ & $92.9 \%$ & $73.1 \%$ & $82.8 \%$ \\
\hline GU246288 vs. JQ695935 & $92.5 \%$ & $98.7 \%$ & $52.8 \%$ & $79.4 \%$ \\
\hline GU246288 vs. GU246296 & $96.9 \%$ & $98.1 \%$ & $87.4 \%$ & $93.9 \%$ \\
\hline GU246287 vs. JQ695935 & $83.9 \%$ & $92.4 \%$ & $48.1 \%$ & $73.3 \%$ \\
\hline GU246287 vs. GU246296 & $86.9 \%$ & $91.7 \%$ & $76.4 \%$ & $84.6 \%$ \\
\hline JQ695935 vs. GU246296 & $95.6 \%$ & $99.4 \%$ & $54.6 \%$ & $81.7 \%$ \\
\hline
\end{tabular}

Note: The sequence alignment analyses for the entire ITS1-5.8S-ITS2 sequences and segmented sequences were performed using Vector NTI Advance 9 (Invitrogen) based on the O. sinensis sequences registered in GenBank, according to the segmentation annotations released by GenBank. GenBank did not provide segmentation information for nrDNA sequence JQ695935 (Genotype \#11). The segmentations for JQ695935 are based on the alignment to the sequence segments of AB067721. 
Table 3 Percentages of AT residues in the ITS segments of Genotype \#1 (the GC-biased AB067721) of Category 1, Genotypes \#7-\#11 of the $O$. sinensis transversion mutants (AJ488254, GU246286, GU246288, GU246287 and JQ695935) of Category 2, and Genotype-\#12 of the Insertion/Deletion mutants (GU246280 and GU246296) of Category 3.

\begin{tabular}{|c|c|c|c|c|c|c|c|c|c|}
\hline \multirow[t]{2}{*}{ Genotype } & \multirow{2}{*}{$\begin{array}{c}\text { GenBank } \\
\text { Accession } \\
\#\end{array}$} & \multicolumn{2}{|c|}{ Residues } & \multirow{2}{*}{$\begin{array}{l}\text { AT }(\%) \\
\text { of total }\end{array}$} & \multicolumn{3}{|c|}{$\begin{array}{c}\text { \# of mutant bases } v s . \\
\text { AB067721 }\end{array}$} & \multicolumn{2}{|c|}{ Ratio of } \\
\hline & & AT & GC & & In./De. & Transv. & Transit. & $\begin{array}{c}\text { Transv. vs. } \\
\text { Transit. }\end{array}$ & $\begin{array}{c}\text { In./De. } \\
\text { vs.Transv./Trans } \\
\text { it. } \\
\end{array}$ \\
\hline$\# 1$ & AB067721 & 264 & 413 & $39.0 \%$ & & & & & \\
\hline \#7 & AJ488254 & 184 & 280 & $34.7 \%$ & 4 & 9 & 10 & 0.90 & 0.21 \\
\hline$\# 8$ & GU246286 & 216 & 318 & $40.5 \%$ & 4 & 25 & 27 & 0.93 & 0.08 \\
\hline$\# 9$ & GU246288 & 190 & 344 & $35.6 \%$ & 8 & 11 & 7 & 1.57 & 0.44 \\
\hline$\# 10$ & GU246287 & 211 & 314 & $40.2 \%$ & 12 & 51 & 29 & 1.76 & 0.15 \\
\hline$\# 11$ & JQ695935 & 272 & 350 & $43.7 \%$ & 29 & 75 & 40 & 1.88 & 0.25 \\
\hline \multirow{2}{*}{$\# 12$} & GU246296 & 203 & 357 & $36.3 \%$ & 17 & 8 & 5 & 1.60 & 1.31 \\
\hline & GU246280 & 205 & 262 & $36.2 \%$ & 24 & 3 & 4 & 0.75 & 3.43 \\
\hline
\end{tabular}

Note: Alignment analyses were performed using the "discontinuous megablast" algorithm in NCBI GenBank Blast. "In./De." refers to the sum of insertion/deletion mutation bases; "Transv." refers to transversion mutation bases; and "Transit." refers to transition mutation bases.

A BLAST search of the GenBank database identified 5 GC-biased transversion mutant genotypes (AT $=34.7 \%-43.7 \%)$ in Category 2 ( $c f$. Table 2). In ascending order of the mutant base ratios of the transversion vs. transition point mutations, as shown in the upper panel of Table 3, they are Genotype \#7, represented by AJ488254; Genotype \#8, represented by GU246286; Genotype \#9, represented by GU246288; Genotype \#10, represented by GU246287; and Genotype \#11, represented by JQ695935. In contrast with the multiple transition point mutations in Genotypes \#3-\#6, Genotypes \#7-\#11 contain multiple, scattered transversion point mutations with some transition point mutations, and the ratios of transversion mutant alleles to transition mutant alleles were 0.90 (9:10), 0.93 (25:27), 1.57 (11:7), 1.76 (51:29), and 1.88 (75:40), respectively ( $c f$. Table 3$)$. Each genotype displays low overall similarity (81.6\%-95.3\%) with Genotype \#1 ITS1-5.8S-ITS2 segments or $55.1 \%-91.5 \%$ similarity with the ITS2 segments of Genotype \#1 ( $c f$. Table 2). These transversion mutants have insertion/deletion $v s$. transversion/transition mutant base ratios of $<0.5$, distinct from Genotype \#12, with a mutant base ratio $>1.0(c f$. Table 3$)$. 
AB067721

AJ 488254 RC

GU246286

GU246288

GU246287

JQ695935

GU246296

GU246280

AB067721

AJ 488254 RC

GU246286

GU246288

GU2 46287

JQ695935

GU246296

GU246280

AB067721

AJ 488254 RC

GU246286

GU2 46288

GU246287

JQ695935

GU246296

GU246280

AB0 67721

AJ 488254 RC

GU246286

GU246288

GU246287

J0695935

GU246296

GU246280

AB067721

AJ488254 RC

GU246286

GU246288

GU2 46287

JQ695935

GU246296

GU246280

AB067721

AJ488254 RC

GU246286

GU246288

GU246287

JQ695935

GU246296

GU246280

AB0 67721

AJ 488254 RC

GU246286

GU246288

GU246287

JQ695935

GU246296

GU246280

(1) $\mathrm{T}$

(1)

(1)

(1)

(1)

(1)

(1)

101

99) GTTGCCTCGGCGGGACCGCCCCGGCGCCCCAGGGCCCGGACCAGGGCGCCCGCCGGAGGACCCCCAGACCCTCCTGTCGCAGTGGCATCTCTCA GTCAA

(49)

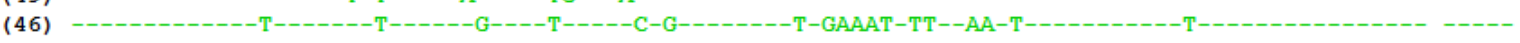

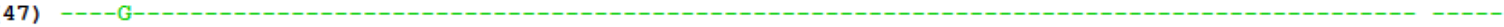

(45) T----T-----TTTG--

(41) --

(46)

(46)

201

198) GAAGCAAGCAAATGAATCAAAACTTTCAACAACGGATCTCTTGGTTCTGGCATCGATGAAGAACGCAGCGAAATGCGATAAGTAATGTGAATTGCAGAAT

(147) -- -

(145)

(146)

(143)

(140)

(145)

(146)

301

(298) TCAGTGAACCATCGAATCTTTGAACGCACATTGCGCCCG CCAGCACTCTGGCGGGCATGCCTGTCCGAGCGTCATCTCAACCCTCGAGCCCCCCGCCTC

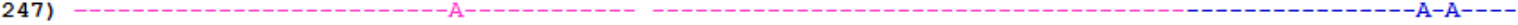

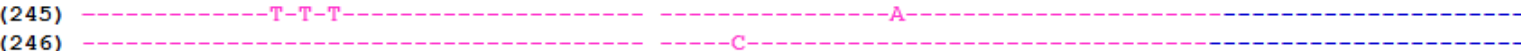

(243)

243)

(240)

(245)

(245)

401

(397) GCGGC GGC GGGGCCC GGCCTTGGGGGTCACGG CCCCGCG CCGCCCCCTAAA CGCA GTGGCGACCCC GCCGC GGCTCCCCTGCGCAG

(346) T---A-T--- ------- -------------- ------

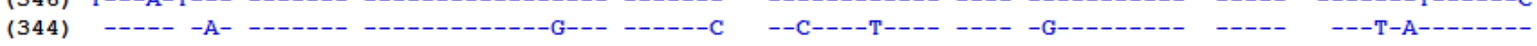

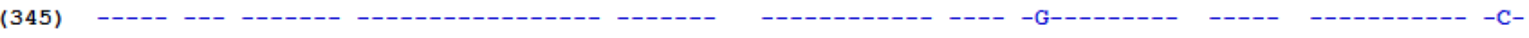

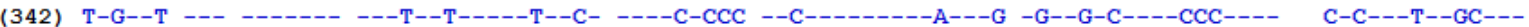

(339) ---- -- -----T TCT-----TT---CTTTATTGAT-ACGG-TTGTA-T-GG- G-TC ---C--G-T-TCG--TC- ------T---ACTT-

(344) ----- --- -------C---------------C----C--C ------------A---G -G--------CG--G-- ------------G-

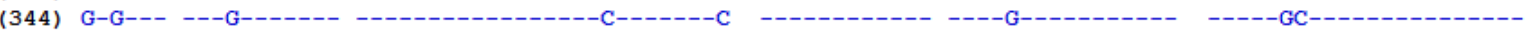

501

600

(483) TAGCTCG CTGAG AACCT CGC ACCGG AG CGCGGAGGCGG TCACG CCG TG AAACCA CCACA CCCT CCAGTTG ACCTCGGATCA G (434) -----G- ---- ---- TC- ---- --G - AA

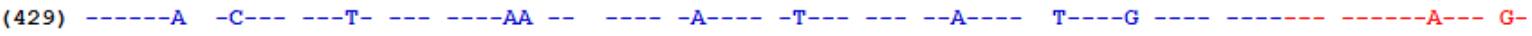

(430) G-A---A -C--- -- -- -- -- -C-- --G --

(433) --TT-AA -C-G- ----C T-G C--C--A--G G-G--GA-G--GGGC-- ---G- A----- C--T-GC---- ------TG--T-G-AT-- G-

(429) C- -TA G--CT ---G-TAC- GG---- TT -----TGATAAT-AT-TG-- T-

(436) ------AAC-GA-- ----- --- ------ --GC----------G-----C--- --A------C----GC-----T------G-----------G-

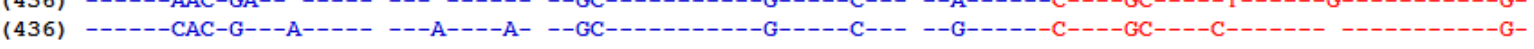

601

GGGA TA CCCGC TGAA CTTAA GCATATCAATAAGCC

(464)

(511) $--\mathrm{G}---\quad-\mathrm{T}---------------$

(510) --- $----\quad--\quad--\quad--\quad---\quad------$

(520) --- ---- -- --- C- ----A----- --

(506) --TC -- - - - - - - - -- TTC-TG-A-TC-G-CG-AG-ATC-GT-GGA-T--CC-CTGAA-TTA--C-TATCAATAA-CG-A--AT-ATAA

(528) ---G----T-- ----- ---- ----- --------

(531) --- G---- --C-----T---- -----G--------

Figure 4 ITS sequence alignment of the 5 groups of $O$. sinensis transversion mutants and 2 insertion/deletion mutants of $\boldsymbol{O}$. sinensis compared with AB067721, using Vector NTI Advance 9 (Invitrogen). AB067721 represents Genotype \#1, the GC-biased O. sinensis, as the reference sequence. AJ488254 (RC, reverse-complement), GU246286, GU246288, GU246287, and JQ695935 represent Genotypes \#7, \#8, \#9, \#10, \& \#11 of Category 2, the O. sinensis transversion mutants. GU246296 and GU246280 represent Genotype \#12 of Category 3, the insertion/deletion mutants of $O$. sinensis. The residues in red were annotated as $18 \mathrm{~S}\left(5^{\prime}\right.$ end) or $28 \mathrm{~S}$ ( $3^{\prime}$ end) rRNA; the residues in green were annotated as ITS1; the residues in pink were annotated as 5.8S rRNA; and the residues in blue were annotated as ITS2. Hyphens indicate identical bases, and spaces denote unmatched sequence gaps.

Am. J. Biomed. Sci. 2016, 8(2), 123-159; doi: 10.5099/aj160200123 @ 2016 by NWPII. All rights reserved 
Genotype \#12, Category 3, includes 3 GCbiased (AT=36.2\%-36.3\%) sequences (GU246296, GU246280, and GU246291) as of July 2015, and contains many insertion and deletion point mutations (17-24 bases) in the ITS1-5.8S-ITS2 segments ( $c f$. Figure 4, Table 3). These sequences also contain a few transversion or transition point mutations. The insertion/deletion vs. transversion/transition mutant base ratios range from 1.31 to 3.43 . This group of mutants shows high similarity to the ITS1 and 5.8S segment sequences of Genotype \#1 H. sinensis but low similarity to the ITS2 sequence of Genotype \#1 (e.g., $87 \%$ for GU246296 vs. AB067721 ITS2 sequence) ( $c f$. Table 2).

In the scientific literature, a threshold of $97 \%$ is commonly accepted as indicative of homology [25]. The ITS1-5.8S-ITS2 segment sequences (excluding the partial $18 \mathrm{~S}$ and $28 \mathrm{~S}$ segment portions) exhibited overall similarities of $73.3 \%$ to $95.5 \%$ in pair-wise comparisons between Genotype \#1 and Genotypes \#3-\#12 and among the mutant genotypes, except for sequence KP731802, whose position in relation to Genotypes \#5 and \#6 is unclear, as mentioned above ( $c f$. Tables 1-2). The low similarities suggest that these genotypes belong to independent fungi that likely evolved from a common genetic ancestor [13,50,53-54]. An extended BLAST search revealed that these 12 mutant genotypes have high-scoring hits $(80.6 \%$ $100 \%$ ) with over 600 entries of $O$. sinensis, $C$. sinensis, and $H$. sinensis sequences, all under the taxid 72228 in GenBank, excluding those sequences registered with other taxa and indeterminate taxa, labeled "sp.".

Tables 1-2 also provide the segmentation similarities (nrDNA ITS1, 5.8S, and ITS2) among the mutant genotypes. In contrast with the relatively high similarities $(92.9 \%-100 \%)$ for the 5.8S ribosomal RNA genes when comparing Genotype \#1 AB067721 with the GC-biased Genotypes \#3, \#7-\#12, the similarities varied from $86.2 \%$ to $99.4 \%$ for the ITS1 segment and from $55.1 \%$ to $93.0 \%$ for the ITS2 segment ( $c f$. Table 2). The low percentages of AT residues in the ITS sequences of Genotypes \#3, \#7-\#12 are similar to that of Genotype \#1 ( $c f$. Table 3) but are much lower than those $(46.5 \%-55.2 \%)$ for the AT-biased Genotypes \#4-\#6. Alignment with the $A B 067721$ sequence revealed that the insertion/deletion mutations comprise $4,4,8,12$, and 29 bases in the ITS segments of Genotypes \#7-\#11, respectively. However, Genotype \#12 (GU246280, GU246291, and GU246296) features multiple scattered insertion and/or deletion mutations, resulting in insertion/deletion $v s$. transversion/transition point mutation ratios of 1.31-3.43 (cf. Table 3).

As shown in Figure 2, the phylogenetic tree branches into 2 major clades: (1) the GC-biased cluster (including Genotypes \#1, \#3, \#7-\#12 and other fungi) and (2) the AT-biased cluster (including Genotypes \#4-\#6). An "other fungi" clade is situated much closer in phylogenetic distance to the GC-biased $O$. sinensis cluster than the longer phylogenetic distances between the $O$. sinensis clusters for the GC-biases (Genotypes \#1, \#3, \#7-\#12) and AT-biases (Genotypes \#4-\#6). As of July 2015, when the GenBank BLAST search was expanded to 1000 maximum target sequences, the "other fungi" clade comprised at least 37 entries in GenBank, including Chaunopycnis sp., Elaphocordyceps sp., Hirsutella sp., Metacordyceps sp., Ophiocordyceps sp., Polycephalomyces sp., and some unknown fungal species, in addition to those fungal species or OTUs identified from natural $C$. sinensis specimens by Zhang YJ et al. [25,51], Zhang S et al. [29] and Xia et al. [48].

From the above sequence and phylogenetic analyses, none of the 3 hypotheses ( $c f$. Sections 2.1, 2.2, and 2.3) regarding the definition of $O$. sinensis have been scientifically confirmed strictly according to Koch's Postulates; therefore, they should be treated equally scientifically. Based on the complex fungal background of natural $C$. sinensis and the uncertain definition of $O$. sinensis, it is difficult at this time to implement the IMA Amsterdam Declaration $1 \mathrm{~F} 1 \mathrm{~N}$ rule because $O$. sinensis may not be a single fungal species (see further discussion below) $[4,13,28-30,39-41,50]$. We hope that the International Commission on the Taxonomy of Fungi (ICTF) will soon establish mechanisms and procedures for handling academic disputes or a committee for fungi, as promised in the 
Amsterdam Declaration signed by 88 IMA members, providing for fair exchange of opinion and a platform for disputing controversies [3941]. In addition to the indiscriminate use of the term $O$. sinensis for both the wild product of the insect-fungi complex and the teleomorphholomorph of the fungus/fungi, as discussed above, the implementation of the $1 \mathrm{~F} 1 \mathrm{~N}$ rule in natural $C$. sinensis research at this time might create further confusion through the indiscriminate use of the term $O$. sinensis for multiple anamorphic fungi ( $c f$. Sections 2.2 and 2.3 and Figure 2). For instance, $\mathrm{Hu}$ et al. [16] described their study material as $O$. sinensis and did not clarify in the manuscript whether the sample was the natural $C$. sinensis insect-fungi complex, teleomorphic $O$. sinensis fungi, or anamorphic $O$. sinensis fungi, nor was it clear which transition, transversion and insertion/deletion mutant genotypes of $O$. sinensis fungi were studied. It is critical for readers to understand this uncertainty in the study materials, results and conclusion, which was clarified by the sponsoring author of that study only upon questioning at a scientific conference. The genomic DNA sample isolated from the $H$. sinensis Co18 strain through the purification of single conidia (asexual form of spores) presented a feature of monokaryosis [16]; this monokaryotic genome differs completely from the genome isolated from the mycelial cultures derived from single ascospores (sexual form of spores); these cultures were likely heterokaryotic and mono-/bi-/trinucleate [52,57] (additional discussion below). In addition, Liu et al. [9] published a study entitled "Transcriptome sequencing and analysis of the entomopathogenic fungus Hirsutella sinensis isolated from Ophiocordyceps sinensis", where the term $O$. sinensis was clearly referring not to the teleomorphic fungus but to the wild product and where the anamorphic name $H$. sinensis could not simply be replaced with the teleomorphic name $O$. sinensis, lest confusion be caused by incorrectly stating " $O$. sinensis isolated from $O$. sinensis". Many other studies have used the same term, either $C$. sinensis or $O$. sinensis, to refer to the wild product, teleomorph/holomorph of $O$. sinensis fungus/fungi, and anamorph of $O$. sinensis fungus/fungi in different contexts, resulting in different and incongruous understandings and interpretations. In addition to the academic confusion regarding the taxonomy, the enforcement of the $1 \mathrm{~F} 1 \mathrm{~N}$ rule in $C$. sinensis research is currently supported only the first hypothesis ( $c f$. Section 2.1) and is inconsistent with and therefore rejects the other 2 hypotheses (cf. Sections 2.2 and 2.3); however, this conclusion is unfortunately not based on experimental evidence. Therefore, the indiscriminate use of the same Latin name for anamorphic fungi, teleomorphic fungi and wild $C$. sinensis of the insect-fungi complex violates the principles of Latin nomenclature and the principle of academic fairness and will continue to cause confusion in academic publications, in annotations in the INSD (including GenBank), and even in governmental documents for regulation of marketed products.

\section{Genetic heterogeneity of natural $C$. sinensis}

The isolation of $C$. sinensis-related fungi from natural $C$. sinensis specimens collected from different geographical areas resulted in the identification of 22 fungal species spanning 13 genera [4,15,17-18]. Using both culturedependent mycological and molecular approaches, Zhang et al. [25] reported the identification of more than 90 fungal species or OTUs from at least 37 genera from the stroma, sclerotia, or external mycelial cortices of natural $C$. sinensis collected from Tibet and Sichuan Provinces. To profile the microbiota of natural $C$. sinensis specimens using real-time PCR and cloningsequencing techniques, Xia et al. [48] identified the dominant fungal species or OTUs from the genera Geomyces, Phoma, and Trichocladium in the caterpillar body and the dominant fungal species or OTUs from the genera Geomyces and Cladosporium in the stroma, in addition to the identification of multiple bacterial species. Microbiota profiling studies by Zhang et al. [25] and Xia et al. [48] using various techniques did not report the detection of Genotype \#1 $H$. sinensis from the caterpillar body and stroma of natural $C$. sinensis specimens, instead suggesting different dominant fungi in the $C$. sinensis 
compartments. Of the over 50 participants in a $C$. sinensis research conference on Oct 29, 2005, 19 scholars reached a hypothetical "consensus" that $H$. sinensis is the sole anamorph of $O$. sinensis based entirely on the compilation of indirect evidence obtained using several techniques $[4,14$ $15,20]$. However, no scientific reports have yet to fully satisfy Koch's Postulates, and no successful artificial production of sexual fruiting bodies and ascospores derived from any of the absolutely pure, postulated anamorphic fungal strains under strict aseptic conditions has been documented $[6,13,15-21,50]$. Unfortunately, the majority of natural fungi still cannot be cultured [23-26], although culture-independent molecular techniques have enabled the identification of greater numbers of fungal taxa and genotypes in natural $C$. sinensis specimens $[8,11-13,26,42,46-$ 49,58-61]. Several studies have also reported dynamic changes in the abundance (reflecting the rates of fungal proliferation, degradation and other biological processes) of $H$. sinensis, other fungi and the mutant genotypes of $O$. sinensis in the different compartments of natural $C$. sinensis during maturation [12-13,26,49,54-57,59-60,62].

Using mycelia from the tissue cultures of the C. sinensis caterpillar body as the study material, Mao et al. [58] reported the detection of significantly larger conidia belonging to Genotype \#4 AB067744 of the AT-biased mutants ( $c f$. Figures $1 \& 2$ ) from $C$. sinensis specimens collected from Deqing in Yunnan Province, and significantly smaller conidia belonging to Genotype \#5 AB067740 (GU233806) of the AT-biased mutants from $C$. sinensis specimens collected from Tibet, Qinghai and Sichuan Provinces. In contrast with the detection of the ITS sequence of Genotype \#1 $\mathrm{H}$. sinensis [14,63-69], the detection of AT-biased Genotypes \#4 and \#5 O. sinensis mutants, but not of Genotype \#1 H. sinensis, by Mao et al. [58] contradicted the hypothesis that $H$. sinensis is the sole anamorph of $O$. sinensis and was inconsistent with the study conclusion of the microcycle conidiation of the $C$. sinensis ascospores [20,70-71] (more discussion below). Non-detection of Genotype \#1 H. sinensis from the stroma and caterpillar body of $C$. sinensis has similarly been reported in other microbiota studies $[25,48]$.

Can these multiple mutant $O$. sinensis fungi be detected from a single set of genomic samples isolated from $C$. sinensis specimens collected from a single production area? In other words, are the PCR ITS amplicons of a single $C$. sinensis genomic DNA sample homogeneous or heterogeneous? In an impure sample pool, such as the $C$. sinensis insect-fungi complex, the dominant DNA components in the pool of amplicons may represent the fungal DNA components with the highest amplification efficiency under particular PCR conditions and may or may not represent the actual dominance of the fungal species in natural $C$. sinensis specimens. The dominant and minor components of the PCR amplicons that can be sequenced may lack secondary structures or specific conformations that cause sequencing failures [26,55-57].

To specifically address the question of amplicon heterogeneity, a dual-step nested-PCR strategy was used, comprising an initial PCR using "universal" primers and a second PCR using inward, fungus-specific primers against the amplicon templates obtained from the first PCR, followed by molecular cloning-sequencing $[49,54]$. The results demonstrated the coexistence of the ITS sequences of the GC-biased $H$. sinensis (Genotype \#1), AT-biased O. sinensis fungi (Genotypes \#4 and \#5), and P. hepiali in the stroma and caterpillar body of both premature and mature $C$. sinensis specimens collected from Sichuan and Qinghai Provinces [49]. Subsequent studies using the same strategy but different fungus-specific primers for the second PCR confirmed the coexistence of the GC- and ATbiased genotypes of $O$. sinensis fungi and $P$. hepiali and uncovered additional AT-biased genotypes of $O$. sinensis (Genotype \#6, and sequence KP731802, which is highly homologous to both Genotypes \#5 and \#6; cf. Figures $1 \& 2$ and Table 1) from the caterpillar body, stroma, ascocarp, and ascospore samples of natural $C$. sinensis collected from Sichuan and Qinghai Provinces [11-12,26,50,59-60]. Another study of the cultured mycelia of single-ascospore isolates of $C$. sinensis collected from Qinghai 
Province detected Genotypes \#1 (GC-biased) and \#5 (AT-biased) of culturable $O$. sinensis fungi using genotype-specific primers [57]. Southern blot analysis also confirmed the coexistence of both GC- and AT-biased genotypes of $O$. sinensis fungi and $P$. hepiali ( $c f$. Upper panels of Figure 5) [26,59].

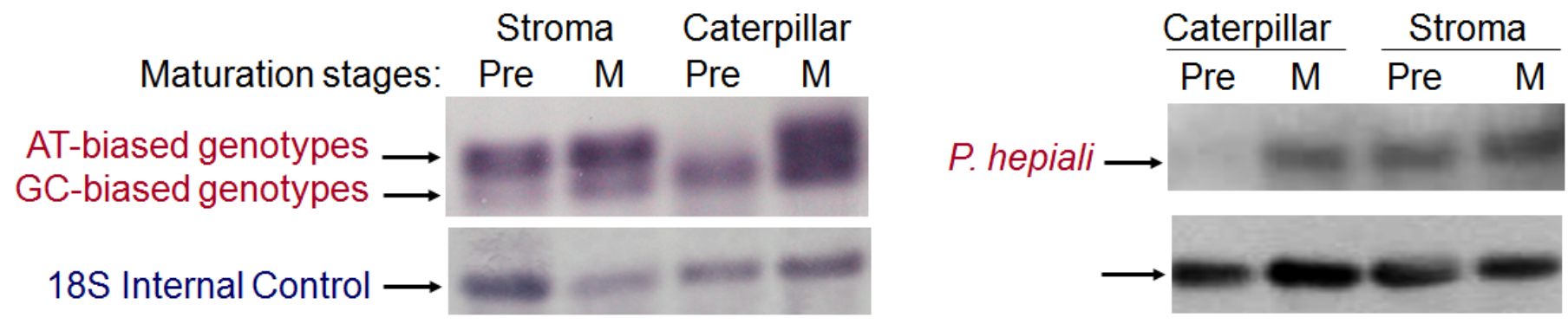

Figure 5 Southern Blot of $C$. sinensis nrDNA in the stroma and caterpillar body of natural $C$. sinensis specimens during maturation. [Reproduced with permission from AJBMS (www.nwpii.com/ajbms) Am J Biomed Sci 2010; 2(3): 217-238] [26].

Upper-Left Panel: Using $H$. sinensis-specific probe for Southern blotting. Upper-Right Panel: Using P. hepialispecific probe for blotting. Both Lower Panels: Using nonspecific 18S internal control probe for blotting. PreM, premature $C$. sinensis; $\mathrm{M}$, mature $C$. sinensis.

To further confirm the coexistence of multiple genotypesof $O$. sinensis fungi, a biochip-based MassARRAY single nucleotide polymorphism (SNP) matrix-assisted laser desorption/ionization-time of flight (MALDITOF) mass spectrometry (MS) technique was used to genotype the natural $C$. sinensis specimens using several extension primers specific to SNP alleles in the sequences of $O$. sinensis genotypes. When the premature stroma of $C$. sinensis collected from Sichuan Province were examined, the coexistence of 2-4 SNP alleles was reported, representing both transition and transversion point mutations, at each of several SNP sites $[26,55]$. As shown in the upper panel of Figure 6, using the extension primer 067721477 [26], Allele G represents the GC-biased Genotypes \#1-\#3, \#7-\#9, and \#11-\#12; Allele A represents the AT-biased Genotypes \#4-\#6, with a single-base $\mathrm{G} \rightarrow \mathrm{A}$ transition mutation (cf. Figure 1); and Alleles $\mathrm{C}$ and $\mathrm{T}$ represent the single $\mathrm{G} \rightarrow \mathrm{C}$ and $\mathrm{G} \rightarrow \mathrm{T}$ transversion mutations, possibly in Genotype \#11
JQ695935 and Genotype \#10 GU246287, respectively.

As shown in the lower panel of Figure 6, using the extension primer 067740-328 [26], Allele A (reverse-complement sequence; "T" at nucleotide 328 in the AB067740 sequence shown in Figure 1) represents Genotypes \#5 and \#6 (the AB067740 and KJ720572 sequences); Allele G (reverse-complement nucleotide; "C" shown in Figure 1) represents Genotype \#4 AB067744; and Alleles T and C represent 2 unknown transversion mutant genotypes. Additional SNP MS analysis (not shown in this review) identified a $G \rightarrow T$ transversion mutant SNP using extension primer 067721-531, corresponding to Genotype \#10 GU246287; a $\mathrm{C} \rightarrow \mathrm{A}$ transversion mutant SNP using extension primer 067740-324, corresponding to Genotype \#7 AJ488254; and a $\mathrm{G} \rightarrow \mathrm{T}$ transversion mutant SNP using extension primer 067740-360, corresponding to Genotype \#10 GU246287 [12,26,55-56,60]. 

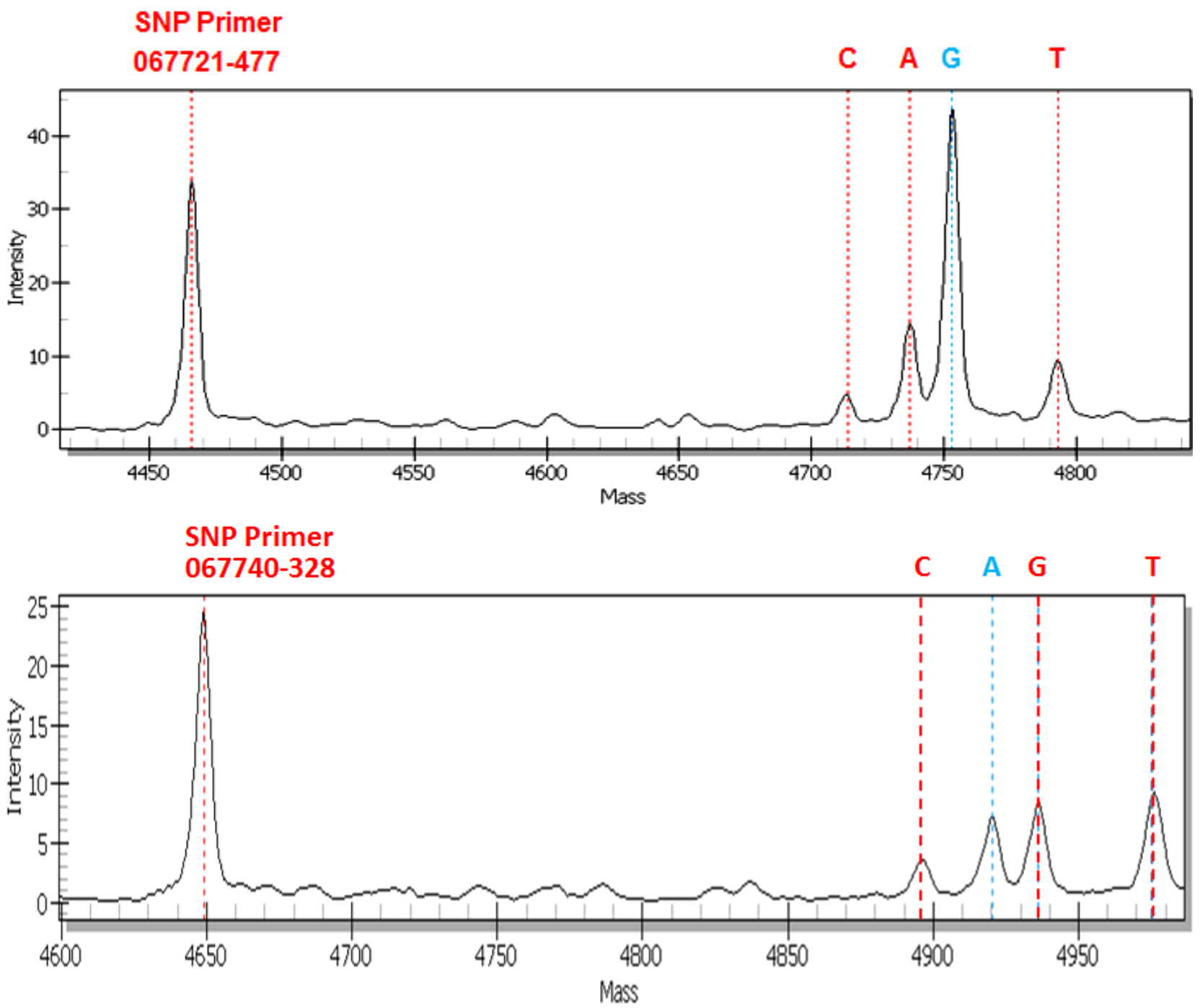

Figure 6. MassARRAY SNP MALDI-TOF mass spectra [Reproduced with permission from AJBMS (www.nwpii.com/ajbms) Am J Biomed Sci 2010; 2(3): 217-238] [26].

Upper panel: The SNP extension primer 067721-477 was used to distinguish between the GC- and AT-biased genotypes and the transversion mutation genotypes of $O$. sinensis. The extension reaction template was the amplicon from PCR with the "universal" primer pair ITS5/4. The 067721-477 primer was extended to the SNP at position 477 in the AB067721 sequence ( $c f$. Figures $1 \& 4$ for the allele location). Allele "G" represents GC-biased Genotypes \#1-\#3, $\# 7-\# 10$, and \#12 (cf. Figures $2 \& 4$ ) and is the result of a primer extension reaction with an extended guanine. Allele "A" indicates Genotypes \#4-\#6 (the AT-biased genotypes) and was the result of a primer extension reaction with an extended adenine. Alleles " $\mathrm{C}$ " and " $\mathrm{T}$ " are the results of primer extension reactions with an extended cytosine or thymine, representing Genotype \#11 JQ695935 with a $\mathrm{G} \rightarrow \mathrm{C}$ transversion point mutation, and Genotype \#10 GU246687 with a $\mathrm{G} \rightarrow \mathrm{T}$ transversion point mutation, respectively.

Lower panel: The SNP extension primer 067740-328 was used to distinguish between the AT-biased genotypes of $O$. sinensis and the unknown transversion mutation genotypes. The extension reaction template was the PCR amplicon obtained using the AT-biased $O$. sinensis-specific primer pair HsATp1/2. The SNP extension primer 067740-328 was extended to the SNP at position 328 in the reverse-complement sequence of AB067740 ( $c f$. Figure 1 for the allele location). Allele "G" (reverse-complement; Allele "C" at the position 328 in the AB067744 sequence shown in Figure 1) represents Genotype \#4 AB067744 and was the result of a primer extension reaction with an extended guanine. Allele "A" represents the AB067740 and KJ720572 sequences (Genotypes \#5 and \#6) and is the result of a primer extension reaction with an extended adenine. Allele " $\mathrm{C}$ " and " $\mathrm{T}$ " denote 2 transversion mutant genotypes of unknown sequences and are the results of primer extension reactions with an extended cytosine or thymine, respectively. 
The coexistence of multiple transition, transversion and insertion/deletion mutant genotypes was observed under dynamic maturational alterations. Upon the maturation of natural $C$. sinensis, the MS peak heights of some alleles were higher or lower, and some alleles disappeared [12,55-56]. Restriction fragment length polymorphism (RFLP), cloning-sequencing, SNP MS genotyping, and Southern blotting revealed a potential predominance of Genotype \#4 (AB067744) in the early development of $C$. sinensis stroma, as well as the $C$. sinensis ascocarps, whereas Genotypes \#5 and \#6 (AB067740 and KJ720572) of the AT-biased $O$. sinensis species dominated the mature stroma of $C$. sinensis and the ascospores [12,26,56,60]. Li Yi et al. [57] reported the successful detection of Group C (Genotype \#5) ITS sequences and the unsuccessful detection of Group B (Genotype \#4) ITS sequences in the mycelial culture of the wild-type ascospores of $C$. sinensis, in addition to the detection of Genotype \#1 AB067721. The aforementioned dynamic maturational alterations were observed in studies of $C$. sinensis specimens collected from a single production area [12,56-57,60], whereas a geographical distribution pattern of the AT-biased genotypes, associated with conidia of various sizes, was observed using tissue cultures of the caterpillar bodies of $C$. sinensis (unfortunately, no clear descriptions of the maturation stages of the natural specimens were given) [58].

The heterogeneity of the PCR amplicons can also be viewed in an endonuclease digestion assay $[12,26]$. For instance, the GCbiased sequences (Genotypes \#1-\#3 and \#7$\# 12$; cf. Figure 1) contain an EcoRI site (GAATTC) at nucleotides 294-299 in the Genotype \#1 AB067721 sequence ( $c f$. Figures $1 \& 4)$. This restriction site is lost in the ATbiased sequences (Genotypes \#4-\#6; $c f$. Figure 1), reflecting a single $\mathrm{C} \rightarrow \mathrm{T}$ transition mutation to GAATTT at the bases allelic to position 299 in the AB067721 sequence. Using a pair of Hsprp1/3 primers that are specific for Genotype \#1 H. sinensis, the 4
PCR amplicons were amplified from the premature stroma of $C$. sinensis as shown in the left panel of Figure 3, in which the 440(A)-bp amplicon moiety predominates [26]. After overnight incubation with EcoRI, digestion of trace amounts of the recovered 440(A)-bp amplicons was observed, apparent as two faint fragments (200- and 240-bp) in the right panel of Figure 3, representing Genotypes \#1-\#3 and \#7-\#12. The vast majority of the recovered 440(A)-bp amplicons were EcoRI-resistant, apparent as the 440 (B)-bp DNA moiety in the right panel of Figure 3, representing the AT-biased Genotypes \#4-\#6, which are the dominant components of the heterogeneous 440(A) amplicons [26]. Although Genotype \#1 (the 200- and 240-bp fragments in the right panel of Figure 3) and Genotype \#2 (the 320-bp amplicon in the left panel of Figure 3) are both GC-biased [26], these 2 genotype moieties display distinct development patterns during $C$. sinensis maturation [12]. The results of the EcoRI digestion RFLP assay ( $c f$. Figure 3) and Southern blot assay (cf. Figure 5) demonstrated that GC-biased $H$. sinensis (Genotype \#1) is a minor component of the heterogeneous genomic DNA pool in the stroma of $C$. sinensis, with or without using PCR amplification, although the relative quantity of the GC-biased genotypes, including Genotype \#1 H. sinensis, showed a slight increase during $C$. sinensis maturation [12,26,59]. A study using real-time qPCR and amplicon cloning profiled the microbiota of natural $C$. sinensis specimens and detected ITS sequences of Genotype \#1 in 2 amplicon clones out of a total of 43 clones from the external mycelial cortices of natural $C$. sinensis specimens, but none were detected from the specimens of the stroma (41 clones) or caterpillar body (40 clones) of $C$. sinensis [48]. Of the 97 sequences submitted to GenBank, the ITS sequences of the other 95 clones belong to other fungi or undetermined fungi, in addition to the 97 bacterial $16 \mathrm{~S}$ sequences submitted to GenBank. To investigate the fungal infection pathways, Lei et al. [72] also used real-time qPCR to 
quantify the fungal species existing in the tissues (including body wall, fat body, hemolymph and intestinal wall) of host Thitarodes larvae. However, the primers that they designed (IF2/IR2) had extremely high homology with the ITS1-5.8S segment sequences of Genotypes \#1-\#3 and \#7-\#12 of GC-biased $O$. sinensis, leading to nonconclusive results for the biomass quantifications of the multiple fungal species.

The conservation of $5.8 \mathrm{~S}$ nrDNA in fungal species over evolutionary time scales has been hypothesized, and the large sequence variation $(85 \%-89 \%$ similarity; $c f$. Table 1) in the 5.8S segment between the GCbiased Genotype \#1 and the AT-biased genotypes "far exceeds what is normally observed in fungi, even at higher taxonomic levels (genera and families)" [26,53,55-56]. The 5.8S segment was $92 \%-97 \%$ similar among the AT-biased genotypes ( $c f$. Table 1). Accelerated evolution (92.9\% and $94.8 \%$ ) in the 5.8S genes proposed by Stensrud et al. [53] can also be observed between Genotype \#1 and the transversion mutation Genotypes \#8 and $\# 10$, respectively ( $c f$. Table 2 ), but the 5.8S sequences of Genotypes \#7, \#9, and \#11$\# 12$ are highly conserved (>98\%). Taking advantage of the low similarity of the $5.8 \mathrm{~S}$ segment amongst the AT-biased genotypes, as shown in the lower panel of Figure 6, SNP MS genotyping using extension primers 067740-328 distinguished between Genotype \#4 AB067744 and Genotypes \#5/\#6 AB067740/KJ720572 from the heterogeneous PCR amplicon pool amplified from the genomic DNA samples of the $C$. sinensis stroma collected from Sichuan Province $[12,26,55-56,60]$. In addition, the 2 transversion mutant SNP alleles (Alleles T and C) shown in the lower panel of Figure 6 did not match any of the known transversion mutant genotype sequences described in this review, indicating the heterogeneous coexistence of at least 2 additional unknown transversion mutant genotypes in the PCR amplicon pool from a single $C$. sinensis genomic DNA sample. Using the extension primer 067740-324, in addition to the detection of Allele $\mathrm{T}$ for Genotype \#4 AB067744 and Allele $C$ for Genotype \#5 AB067740, Allele A was also detected with a high MS peak height [26], probably representing the $\mathrm{C} \rightarrow \mathrm{A}$ transversion mutation at nucleotide 273 in the Genotype \#7 AJ488254 sequence ( $c f$. Figure 4). A low MS peak height of Allele $\mathrm{T}$ was detected at position 360 in the Genotype \#5 AB067740 sequence using the extension primer 067740360 [56], probably representing the $\mathrm{G} \rightarrow \mathrm{T}$ transversion mutation at nucleotide 306 in the Genotype \#10 GU246287 sequence; the MS peak height of this allele significantly increased in the stroma with $C$. sinensis maturation. Future studies will likely uncover additional heterogenetic information and identify more transversion mutants in natural C. sinensis specimens.

The above literature review clearly demonstrates the genetic heterogeneity of natural $C$. sinensis specimens and the molecular heterogeneity of the PCR amplicons using the "universal" primers and primers specific to GC-biased Genotype \#1 or AT-biased genotypes, as well as the observation of heterokaryotic structures of the C. sinensis hyphae and ascospores [52,57]. Molecular systematic examination of genetic heterogeneity demonstrated that Genotype \#1 $H$. sinensis is not the dominant fungal species in the compartments of natural $C$. sinensis specimens. Proving that the minor fungal species Genotype \#1 H. sinensis, rather than the major AT-biased species, is the sole anamorph of $O$. sinensis, as hypothesized ( $c f$. Section 2.1), must rely on direct evidence obtained from re-inoculation and re-isolation experiments strictly following Koch's Postulates, using absolutely pure, postulated anamorphic fungus and obtaining the sexual fruiting bodies and ascospores of $C$. sinensis under strictly aseptic experimental conditions. Given the unavailability of the direct evidence to confirm the " $H$. sinensis sole anamorph" hypothesis for $O$. sinensis ( $c f$. Section 2.1), the above literature analysis also indicates that treating the aforementioned mutant genotypes as "incorrect sequences", as 
suggested by Zhang et al. [51], may be neither convincing nor scientifically sound.

\section{Do the multiple $O$. sinensis sequences represent pseudogenes of the $H$. sinensis genome or mutant fungal species independent of the $H$. sinensis genome?}

$\mathrm{Li} \mathrm{Yi}$ et al. [57] sequenced mycelia cultured from wild-type single-ascospore isolates and identified the coexistence of both the Group A (Genotype \#1) GC-biased genotype and Group C (Genotype \#5) of the AT-biased transition mutant genotypes of $O$. sinensis from the genomes of singleascospore isolates; but they were unable to detect any of the 5.8S gene transcripts of the AT-biased genotypes. Therefore, these authors proposed that the highly divergent mutant ITS sequences were ITS pseudogene components of the genome of $H$. sinensis. This ITS pseudogene hypothesis, however, prompted some fundamental questions because the multiple heterogeneous ITS sequences of natural $C$. sinensis specimens were assumed to represent the descendants of a common genetic ancestor during long-term phylogenetic evolution [53]. Are these mutant ITS sequences intra-individual pseudogenic components of the $H$. sinensis genome [57], or do they belong to multiple inter-individual mutant fungal species [51,53-54]? Are the genotypes with multiple transition, transversion, and insertion/deletion point mutations (summarized above) persistently silenced during transcription in $C$. sinensis development under natural conditions, or can Genotype \#5 be counter-silenced during some C. sinensis maturation stages? Goodhead and Darby [73] reviewed the identification and categorization of pseudogenes and suggested "taking the pseudo out of pseudogenes". They defined pseudogenes as junk or relics of genomes and "fragments of once-functional genes that have been silenced by one or more nonsense, frame shift or missense mutations" in the functional homologs. Increasing evidence indicates that some persistent pseudogenes are transcriptionally "alive" and play special roles, undergoing positive selection to persist during evolution, thereby raising questions regarding the ambiguous boundary between genes and pseudogenes and calling for superior nomenclature to replace the term "pseudogenes" and better describe their functions [73-80]. Given the controversy surrounding the concept of pseudogenes, the following conclusions regarding natural $C$. sinensis specimens and the genomes of $O$. sinensis fungi are notable.

\subsection{The functional genes and their "ITS pseudogene" counterparts belong to the genomes of independent fungal species}

Based on the findings of the coexistence of the postulated "ITS pseudogenes" (ATbiased Genotype \#5) with functional copies (GC-biased Genotype \#1) in the genomes of $O$. sinensis single-ascospore isolates, $\mathrm{Li} \mathrm{Yi}$ et al. [57] commented that the hypotheses suggesting that AT-biased ITS sequences represent cryptic (phylogenetic) species [53], different fungal species [54], or different genotypes [26] were all "inaccurate". In contrast, $\mathrm{Li} \mathrm{Yi}$ et al. [57] reported the unsuccessful detection of ITS sequences of Genotype \#4 AB067744 in the same genomic samples using a pair of fungus-specific primers, suggesting that this divergent mutant ITS sequences indeed belong to different fungi. Xiao et al. [54] reported that the mutant genotypes (Groups A, B, and C [53], i.e., Genotypes \#1, \#4, and \#5 in this review) of $O$. sinensis likely belong to independent fungi in natural $C$. sinensis; this conclusion is consistent with the results of Zhang et al. [29], who conducted a bioinformatic analysis of the sequences of $O$. sinensis fungi registered in INSD and used the ITS sequences of the authentic anamorphic $H$. sinensis as an analytical reference. Using various tissue culture samples derived from the caterpillar body of natural $C$. sinensis specimens collected from different geographic areas, Mao et al. [58] reported the detection of sequences of either Genotype \#4 or \#5, but not of Genotype \#1 H. sinensis. These mutant fungi with distinct conidia of significantly 
different sizes most likely exhibited a unique geographic distribution pattern in $C$. sinensis production areas. Thus, the highly variable mutant ITS sequences were attributed to mutant fungal species or OTUs rather than to their coexistence as pseudogenes with functional counterparts in one anamorphic genome.

The existence of multiple inter-individual mutant fungal species is supported by several studies and by genome-wide sequencing of the authentic anamorphic fungus $H$. sinensis $[12,16,26,29,49,53-54,58]$. Although genomewide sequencing of the authentic anamorphic H. sinensis Co18 strain has identified 3 types of pseudogenes, Hu et al. [16] did not report the presence of transition, transversion or insertion/deletion mutant ITS sequences in the whole-genome sequence ANOV00000000 of the $H$. sinensis Co18 strain. Alignment analysis of the $H$. sinensis genome sequence ANOV00000000 further revealed a DNA segment within the 2626-bp sequence KE659721 (=ANOV01021709) that was 99.7\% homologous to Genotype \#1 AB067721 of $O$. sinensis; no other segment sequences with high-similarity homologs were identified within ANOV00000000. The ITS sequences of $O$. sinensis Genotypes \#3-\#12 exhibited $83.0 \%-95.1 \%$ similarity to KE659721, suggesting that the mutant ITS sequences were clearly independent of the whole genome of the anamorphic $H$. sinensis. These results suggest that the multiple point mutations in the $O$. sinensis ITS sequences represent genomes of separate anamorphic fungal species $[16,29,54,58]$, which is, unfortunately, inconsistent with the ITS pseudogene hypothesis of $\mathrm{Li} \mathrm{Yi}$ et al. [57], who studied completely different genomes from that of the Co18 strain of anamorphic $H$. sinensis tested by $\mathrm{Hu}$ et al. [16]. In addition, cloning and sequencing of the ITS amplicons to examine several "pure" anamorphic $H$. sinensis strains revealed the coexistence of $P$. hepiali and $H$. sinensis (Genotype \#1) ITS sequences, but other multiple mutant genotypes of $O$. sinensis (Genotypes \#3-\#12) were not detected [61]. Therefore, these analyses confirmed the conclusion of Xiao et al. [54] that the highly variable mutant ITS sequences belong to different fungi.

Although Li Yi et al. [57] detected only the ITS sequences of Genotypes \#1 AB067721 and \#5 AB067740 and failed to detect the ITS sequences of Genotype \#4 AB067744, no experiments have been designed to examine the other $O$. sinensis genotypes listed in the GenBank database: 3 transition mutant genotypes (Genotypes \#2-\#3 and \#6) of Category 1, 5 transversion mutant genotypes (Genotypes \#7-\#11) of Category 2, and the 3 insertion/deletion mutants within Genotype \#12 of Category 3. The inability to detect Genotype \#4 AB067744 by Li Yi et al. [57] reflected the possibility that Genotype \#4 AB067744 sequences might be associated with a fungus (which might or might not be culturable) in the different compartments of natural $C$. sinensis at different maturation stages $[12,49,56,60]$ or in different geographic production areas $[49,54,58]$. The incomplete study design and findings of the report in question [57] therefore provide insufficient and contradictory evidence supporting the ITS pseudogene hypothesis.

The results of other studies also contradict the assumption of the genomic coexistence of "dysfunctional" ITS pseudogenes and functional counterparts in one genome of pure $O$. sinensis. The combined use of Southern blotting after EcoRI preparation and EcoRI digestion RFLP assays resulted in the detection of a single Southern blot moiety from genomic DNA isolated from a pure $H$. sinensis strain and of a doublet from genomic DNA isolated from the stroma and caterpillar body of natural $C$. sinensis [26]. These results indicate that the EcoRI-resistant AT-biased sequences did not exist in the genome of pure GC-biased $H$. sinensis but rather coexisted with the EcoRIsensitive GC-biased sequences in the fungal genome pool of natural $C$. sinensis. As shown in the upper-left panel of Figure 5, Southern blot analysis of the EcoRI-digested genomic DNA demonstrated that the relative biomasses of the ITS segments of the mutant 
$O$. sinensis genotypes (the faster-migrating GC-biased Genotypes \#1, \#3, and \#7-\#12 vs. the slower-migrating AT-biased Genotypes \#4-\#6 in gel electrophoresis) were dynamically altered in a non-synchronized manner during $C$. sinensis maturation over a short period of time (weeks or 1-2 months) in the stroma and caterpillar body [26,59], although $\mathrm{Li}$ Yi et al. [57] misinterpreted the maturational phenomenon of these asynchronous alterations in the biomasses of $O$. sinensis mutants as the "AT-biased genotypes are not found in the sclerotium of $O$. sinensis". Briefly, in premature $C$. sinensis ( $c f$. upper-left panel of Figure 5), the fungi with the AT-biased genotypes, represented by the slower-migrating DNA moiety, were probably absence in the caterpillar body but highly predominant in the stroma; the biomass greatly increased in both compartments during $C$. sinensis maturation [26,59]. The biomass of the GC-biased genotypes, including Genotype \#1 H. sinensis and Genotypes \#3, \#7-\#12 (the fastermigrating DNA moiety in the upper-left panel of Figure 5), was extremely low in the stroma of premature $C$. sinensis and increased with $C$. sinensis maturation, but was never the predominant DNA species in the stroma. Consistent with the hypothesis of Liang et al. [19] that natural $C$. sinensis is an integrated micro-ecosystem, these asynchronous maturational changes in the biomasses of mutant $O$. sinensis genes do not support the coexistence of multiple $O$. sinensis genotype sequences in the genome of a purified anamorphic $H$. sinensis and are not consistent with the principle of genetic stability or the assumption of the genomic coexistence of the pseudogenic mutant sequences and functional gene counterparts in the genome of Genotype \#1 H. sinensis. These asynchronous maturational alteration phenomena at the genomic DNA level were also confirmed in other experiments by EcoRI digestion RFLP and SNP MS genotyping assays [12,13,26,56].

In direct contrast with the coexistence of mutant "ITS pseudogenes" and functional gene counterparts in the same genome of an anamorphic fungal species, the most reasonable interpretation of the inability to detect Genotype \#4 and other mutant genotypes and of the phenomenon of dynamic, asynchronous changes in the biomasses of mutant genotype species during $C$. sinensis maturation is that these ITS sequences with multiple, scattered transition, transversion, and/or insertion/deletion point mutations belong to the genomes of independent fungi [13,26,50,53-54]. The asynchronous changes in fungal presences in the different compartments of $C$. sinensis during the spring-summer transition likely reflect maturation-related changes in the rates of proliferation, degradation and other biological processes of the different fungi, which may be psychrophilic (associated with the early development of $C$. sinensis in winter and early spring) or mesophilic (associated with the late maturation of $C$. sinensis in late spring and summer).

\subsection{Distinct genome samples tested in different studies}

Previous studies have analyzed truly different genome samples of these organisms: (1) total genomic DNA isolated from a pure, authentic anamorphic $H$. sinensis Co18 strain that was used in the genome-wide sequencing study [16]; and (2) total genomic DNA isolated from $O$. sinensis mycelia derived from a 25-day incubation (in a liquid culture medium) of wild-type isolates from single ascospores of $C$. sinensis, whereas the multicellular teleomorph of $O$. sinensis showed structural features including mono-, bi-, and trinucleate cells in each ascospore $[52,57]$. Compared with the cultured mycelia of the anamorphic $H$. sinensis Co18 strain containing a homogeneous GC-biased Genotype \#1 ITS1-5.8S-ITS2 segment in its genome, the cultured mycelia derived from the heterokaryotic wild-type $C$. sinensis ascospores comprised heterogeneous ITS components: both Group A (Genotype \#1) GC-biased and Group C (Genotype \#5) ATbiased mutant ITS sequences, whereas other mutant ITS sequences (of Genotypes \#2-\#4 
and \#6-\#12) were not detected. Xiao et al. [81] confirmed the overall polymorphic differences between $H$. sinensis strains and the wild-type $C$. sinensis ascospore samples via ISSR molecular marker polymorphism analysis, although the authors incorrectly used density-unweighted algorithms for the similarity computations and phylogenetic tree construction. Do these results suggest that the ascospores of $C$. sinensis were the heterokaryotic meiotic organism if the genomes tested were isolated from the mycelial culture of truly pure singleascospore isolates [2]? Unfortunately, Li Yi et al. [57] did not provide technical information regarding the collection, isolation and purification of their study materials, ascospores and genomes, instead guiding the readers to a Ph.D. dissertation [82] that is unavailable to the general public as a reference.

The distinct genotyping results from the cultured mycelia obtained from wild-type, mono-/bi-/trinucleate single-ascospore cultures [57] and the anamorphic mycelia obtained from a single conidia culture [16] raise a series of legitimate questions. (1) Were the wild-type multicellular, mono-/bi/trinucleate $C$. sinensis single-ascospore samples from homokaryotic or heterokaryotic teleomorphic organisms [2,52]? (2) Was the study material used by Li Yi et al. [57] from an absolutely pure homokaryotic $O$. sinensis fungus or from a mycelial mixture of anamorphic haploid organisms derived from in vitro culture of wild-type polykaryotic, multicellular isolates? (3) Were there two or more sets of chromosomes in the test material examined by Li Yi et al. [57], as indicated by the fluorescent staining showing mono-/bi/trinucleate structures in each multicellular ascospore reported by the same group of researchers [52]? (4) Were the phenotype(s) and genotype(s) of the conidia obtained from the microcycle conidiation of ascospores by Xiao et al. [20], Liu et al. [70] and Mo et al. [71] similar to those of the conidia and mycelia obtained by Li Yi et al. [57] from the culture of $C$. sinensis ascospores? (5) Do the conidia of various sizes and shapes observed in the experimental microcycle conidiation of ascospores and conidia reported by Xiao et al. [20] have different genotypes, similar to those reported by Mao et al. [58]? (6) Which karyotype(s) of cells (mononucleate, binucleate, or trinucleate discovered by Bushley et al. [52]) of the multicellular ascospores are capable of conidiation? (7) Do the larger conidia derived from conidiation of ascospores have the same karyotype(s) and genotypes as their parental wide-type ascospores [52]? (8) Does the conidiation observed in the studies of microcycle conidiation of ascospores represent all natural conidiation processes of all mononucleate, binucleate and trinucleate cells of the wildtype, multicellular, heterokaryotic ascospores of $C$. sinensis $[2,18,21,52,83]$ ? As reported by Mao et al. [58], fungi with Genotypes \#4 \& \#5 exhibit the same mycelial morphology as Genotype \#1 $H$. sinensis, and the fungal conidia may be larger for Genotype \#4 than for Genotype \#5, suggesting the impossibility of distinguishing between the mutant fungi of various genotypes based solely on their microscopic morphology.

\subsection{The silencing of $5.8 S$ genes}

In addition to obtaining both the Genotype \#1 sequence and one (Genotype \#5) of the AT-biased sequences from the genomes of the mycelial culture of the wild-type single-ascospore isolates, $\mathrm{Li} \mathrm{Yi}$ et al. [57] also examined the transcripts of $5.8 \mathrm{~S}$ genes derived from reverse transcription PCR, which resulted in the detection of the $5.8 \mathrm{~S}$ cDNA only for Genotype \#1 (Group A), but not for Genotype \#5 (Group C) or other genotypes. This result provided critical evidence that the 5.8S gene of the GC-biased Genotype \#1 was the functional copy of the $O$. sinensis genome, whereas Genotype \#5 5.8S genes were the dysfunctional, pseudogenic components of the teleomorphic $O$. sinensis genome.

The use of fungal non-specific $5.8 \mathrm{~S}-\mathrm{F} / \mathrm{R}$ primers in the study [57] makes it necessary to seriously consider the extremely high 
similarity between the 5.8S genes of Genotypes \#1-\#3, \#7-\#9, \& \#11-12 (cf. Figures $1 \& 4), P$. hepiali and hundreds of different fungi [26] and the low similarity between the 5.8S genes of the GC-biased Genotype \#1 and AT-biased Genotypes \#4-6 (cf. Figure 1) before concluding that the detected $5.8 \mathrm{~S}$ transcript truly belonged to Genotype \#1 $H$. sinensis and thus that the $5.8 \mathrm{~S}$ transcripts of the mutant genotypes were not detected. Thus, doubt regarding the functionality of the $5.8 \mathrm{~S}$ gene of the GCbiased Genotype \#1 needs to be further addressed if the detected 5.8S cDNA did truly belong to Genotype \#1 of $O$. sinensis, as stated in [57], because the authors did not disclose either the experimental design or the results for the detection of other fungi and because the ITS sequences of $H$. sinensis and $P$. hepiali were detected using genomic DNA isolated from the ascospores of natural $C$. sinensis [60]. To confirm that the detected $5.8 \mathrm{~S}$ cDNA truly belonged to Genotype \#1 of $O$. sinensis, the combined use of other molecular techniques may be warranted.

Before concluding that the AT-biased 5.8S genes are non-functional based on the non-detection of AT-biased 5.8S cDNA [57], several other concerns should be addressed:

(1) The homology of the primers, $5.8 \mathrm{~S}-\mathrm{F}$ and $5.8 \mathrm{~S}-\mathrm{R}$, for detecting $5.8 \mathrm{~S}$ cDNA must be verified to ensure the same high primerbinding capability for all AT-biased mutant genotypes under the experimental conditions of the competitive binding of primers to the available templates and to eliminate the potential technical failure of reverse transcription PCR.

(2) Because Li Yi et al. [57] did not detect the ITS sequences for mutant genotypes other than Genotypes \#1 and \#5 from the genomic DNA pool of the singleascospore cultures, it may be assumed that the 5.8S transcripts of Genotypes \#3-\#4 and \#6\#12 cannot be detected from the total RNA pool after being cultured in a liquid culture medium at $18^{\circ} \mathrm{C}$ for 25 days. Claiming that the $5.8 \mathrm{~S}$ genes of those mutant genotypes (Genotypes \#3-\#4 and \#6-\#12) are non- functional pseudogenes would be an overinterpretation.

(3) To confirm the non-detection of the $5.8 \mathrm{~S}$ transcripts of the mutant genotypes of $O$. sinensis, the 3 types of secondary steric conformations of $5.8 \mathrm{~S}$ genes predicted by $\mathrm{Li}$ $\mathrm{Yi}$ et al. [57] for Groups A, B, and C (Genotypes \#1, \#4 \& \#5) of $O$. sinensis must be considered during the primer design because these secondary structures might have a considerable impact on reverse transcription PCR. Multiple sets of primers or other molecular techniques and functional assays may be required in designing a study to examine the 5.8S gene transcription.

(4) $\mathrm{Li} \mathrm{Yi}$ et al. [57] disclosed that teleomorphic ascospores were subjected to liquid-phase rotary-shaker incubation (100 rpm) at $18^{\circ} \mathrm{C}$ in a particular culture medium (PDA with $5 \%$ wheat bran and $0.5 \%$ peptone) for 25 days prior to extraction of total genomic DNA and total RNA from the mycelia. These non-natural 25-day culture conditions might significantly impact the transcription of many functional genes, potentially non-physiologically switching on or off some genes, as was confirmed by the gradual nonlinear reduction in the total number of transcriptomic unigenes from 25,511 after 3 days of fermentation to 25,214 at Day 6 and then drastically down to 16,245 at Day 9 during continuous liquid fermentation [9].

(5) The findings of $\mathrm{Li} \mathrm{Yi}$ et al. [57] are inconclusive if the $5.8 \mathrm{~S}$ genes of the mutant $O$. sinensis genotypes are transcriptionally silenced during some physiological stages of the development and maturation of natural $C$. sinensis, for instance, becoming silenced after the ejection of ascospores. Evidence supporting such dynamic, physiological alterations of gene transcription came from proteomic profiling analysis, indicating significant changes in proteomic profiles in the different compartments of natural $C$. sinensis specimens during maturation [10]. Conditional and periodic silencing of the $5.8 \mathrm{~S}$ genes of mutant genotype fungi must be 
further explored before declaring some genes to be non-functional "pseudogenes".

Natural $C$. sinensis is an insect-fungi complex with an extremely complex life cycle. Studies have reported that the presence/absence and quantities of multiple mutant fungi resulting from fungal proliferation, degradation and other biological processes are altered in different compartments (caterpillar body, stroma, ascocarp and ascospores) of natural $C$. sinensis during different developmental and maturation stages [11-13,19,26,29,49-50,5456,59-60]. Notably, the transcription of the genomes of the intrinsic psychrophilic and mesophilic fungi might also be altered during various stages of $C$. sinensis formation, development, and maturation in the compartments of $C$. sinensis in response to seasonal changes in alpine environmental conditions. Transcriptomic alterations have been demonstrated in 3-, 6-, and 9-day mycelial cultures of a wild-type " $H$. sinensis" L0106 strain isolated from premature natural $C$. sinensis and cultured in vitro in 200-liter submerged stirred fermenters [9]. A proteomic polymorphism study demonstrated dramatic differences in proteome expression in the stroma and caterpillar body of natural $C$. sinensis between the premature and mature stages [10]. Additional studies are necessary to determine whether the so-called "ITS pseudogenes" of the multiple mutant genotype fungi are counter-silenced and actively transcribed to play specific biological roles in the $C$. sinensis lifecycle: (1) fungal infection of host larvae of the family Hepialidae and synergy in infection between the fungi with functional ITS genes and those with so-called "ITS pseudogenes"; (2) initial asexual growth of fungi inside the larva; (3) hibernation in the dead larva during the extremely cold winter on the Qinghai-Tibet Plateau; (4) stroma germination; (5) transition of initial anamorphic fungal growth in premature $C$. sinensis to teleomorphic growth in mature $C$. sinensis; (6) changes during the courses of plasmogamy and karyogamy; (7) formation of polyploids and polykaryons; (8) scaffold construction for the $C$. sinensis ascocarps; (9) meiosis of teleomorphic fungal cells; (10) formation and maturation of the multicellular ascospores; (11) natural ejection and/or semi-ejection of the mature ascospores [60]; (12) post-ejection survival of the teleomorphic ascospores in the environment and the transition to the anamorphic stage(s), etc. The complexity of proving or disproving the non-functionality of the $5.8 \mathrm{~S}$ genes of the AT-biased and other mutant genotypes and the ITS pseudogene hypothesis appears to be far beyond the current knowledge of natural $C$. sinensis, multiple mutant $O$. sinensis fungi, and their accompanying fungi.

The analyses and discussions described above suggest that these multiple, scattered transition, transversion, and insertion/deletion mutant genotype sequences are likely the genomic components not of GC-biased $H$. sinensis but of individual mutant fungi. The genetic heterogeneity of single-ascospore isolates may indicate that the ascospores of $C$. sinensis feature mixtures of the mono-, bi-, and trinucleate multicellular structures $[2,13,50,52,57]$. The reported detection of the $5.8 \mathrm{~S}$ transcript through reverse transcription PCR using non-specific primers may or may not belong to the Genotype \#1 H. sinensis, and the non-detection of the mutant $5.8 \mathrm{~S}$ transcripts under the experimental conditions described in [57] provide insufficient evidence to determine functionality. Other techniques are needed to directly examine the functionality of the $5.8 \mathrm{~S}$ genes of the multiple mutant $O$. sinensis fungi in the natural $C$. sinensis samples at different developmental and maturation stages to differentiate between the truly permanent dysfunction of so-called "ITS pseudogenes" and the temporary transcriptional silencing of the $5.8 \mathrm{~S}$ genes in the caterpillar body, stroma, ascocarp, and ascospores of natural $C$. sinensis during development and maturation. 


\section{Heterogeneous ITS amplicons of the $C$. sinensis ascospores and microcycle conidiation}

The ITS sequences of the GC-biased Genotype \#1 (Group A) and the AT-biased Genotype \#5 (Group C) were simultaneously detected in the heterogeneous amplicon pool from the genomic DNA of the mycelial culture of the wild-type ascospores of natural C. sinensis [57], suggesting heterokaryosis of the $C$. sinensis ascospores with mixed mono/bi-/trinucleate structures in each multicellular ascospore [2,13,50,52]. Further genotyping analysis of the ascospores of $C$. sinensis using PCR amplicon cloning-sequencing and SNP MS genotyping techniques detected several fungi [60]: Genotype \#1 $H$. sinensis, Genotype \#5 AT-biased KJ729098 and KM017747 (98\% homologous to AB067740), Genotype \#6 AT-biased KJ720572 (98\% homologous to EU555436), and $P$. hepiali (100\% homologous to EF555097). These studies using PCR amplicon cloningsequencing and SNP genotyping of the wildtype teleomorphic ascospores [60] or PCR amplicon sequencing after mycelial culture of the ascospores [57] revealed a complex genetic background for the $C$. sinensis ascospores, suggesting that the $C$. sinensis ascospores have a heterokaryotic multicellular structure [2,52].

As discussed above, the occurrence of heterogeneous mutant ITS sequences in the genomes of different fungi and the findings of the mixed mono-, bi-, and trinucleate structures in the single multicellular ascospore directed our interest to the microcycle conidiation of ascospores. Jiang and Yao [17] suggested that microcycle conidiation of the $C$. sinensis ascospores is an auxiliary technique and provides only indirect evidence for confirmation of the correct anamorph of $O$. sinensis. Scholars have doubted whether studies of microcycle conidiation of the ascospores of natural $C$. sinensis under specific experimental conditions can completely profile all conidiation of ascospores, particularly for those anamorphic species for which experimental culture and conidiation are difficult to achieve in laboratory settings $[18,21,23,83]$. Studies of microcycle conidiation of $C$. sinensis ascospores reported the detection of only " $H$. sinensis conidia" through microscopic morphological examinations [20,70-71]. However, this morphological assumption of " $H$. sinensis conidia" might be challenged by the following: (1) the distinct morphology of conidia obtained either from the microcycle conidiation of the $C$. sinensis ascospores or of the anamorphic conidia of $H$. sinensis reported by Xiao et al. [20]; (2) the similar conidial morphology of AT-biased genotype fungi and the Genotype \#1 $H$. sinensis according to Mao et al. [58]; (3) the overall polymorphic differences in molecular markers between pure $H$. sinensis strains and the wildtype $C$. sinensis ascospore samples via ISSR polymorphism analysis [81]; and (4) the simultaneous detection of the ITS sequences of the culturable fungi of Genotypes \#1 and \#5 [57] in addition to those of fungi of Genotype \#6 and P. hepiali [60] from the genomes of the ascospores of natural $C$. sinensis with the mixed mono-, bi-, and trinucleate multicellular structures [52]. Unfortunately, none of these microcycle conidiation studies reported the karyotypes and genotypes of the conidia produced from the conidiation of the $C$. sinensis ascospores [20,70-71]. As discussed above, the mutant sequences of multiple $O$. sinensis genotypes are not likely to represent the pseudogenic components of one genome of the single GCbiased $H$. sinensis fungus but instead to belong to different anamorphic fungi that are closely associated with each other either in a fungal (species) complex formed under natural settings or within the mono-/bi/trinucleate heterokaryotic teleomorph of $O$. sinensis [2,21,52]. Given the successful development of methods for culturing fungal species susceptible to difficulties in ascospore germination and experimental culture in laboratory settings $[18,21,83]$, the combination of microcycle conidiation of 
ascospores and proper molecular approaches in future studies may provide critical insight into the conidiation of the ascospores of natural $C$. sinensis and the profile of heterokaryotic fungal molecules.

\section{Proteomic polymorphisms and protein- coding genes of natural $C$. sinensis}

In a symposium on $C$. sinensis anamorphs on Oct 29, 2005, Prof. Liang ZQ suggested using omic technologies to study natural $C$. sinensis for confirmation of the anamorphteleomorph connection for $O$. sinensis fungi. Despite the strong objection from his mycology colleagues at that conference 11 years ago, the omic approaches used in $C$. sinensis studies in recent years have uncovered a substantial amount of scientific information in several publications, ranging from whole genome, transcriptome, and proteome studies to metabolic/chemical fingerprinting and $C$. sinensis microbiota profiling $[9-10,16,25,48,51,84-85]$, greatly enriching our knowledge of natural $C$. sinensis as a holistic insect-fungi complex and of the anamorph-teleomorph connection for $O$. sinensis fungi. For instance, a study of natural C. sinensis reported profound, dynamic, asynchronous changes in proteomic polymorphisms in the stroma and caterpillar body of $C$. sinensis during maturation [10]. In examining the whole genome protein-coding genes of a purified anamorphic $H$. sinensis strain, Hu et al. [16] reported 6,972 proteincoding genes and identified the genes encoding 2,229 protein families through InterProScan analysis, including both actively transcribed and silent genes. Through transcriptome sequencing of total RNA isolated from natural $C$. sinensis collected from Sichuan Province, however, Xiang et al. [85] reported atotal of 34,289 unique sequences with or without gene annotations (including 17,230 singletons and 17,059 contigs) and identified 7,229 unique transcriptomic sequences with functional annotations through InterProScan analysis. Despite the potential maturation-dependent silencing of gene transcription in natural $C$. sinensis, the numbers of actively transcribed genes identified from the natural $C$. sinensis insect-fungi complex [85] were several times greater than the number of genes (both actively expressed and silent) identified from the genome-wide sequencing of a purified anamorphic $H$. sinensis strain [16], which is inconsistent with the sole anamorph hypothesis for $H$. sinensis described above.

In contrast with a previous examination [85] of the transcriptome profile directly from total RNA isolated from natural $C$. sinensis, another transcriptome study [9] was conducted on cultures of a wild-type $H$. sinensis L0106 strain that was isolated from the tissue of the premature $C$. sinensis collected in May from Qinghai Province. The ITS sequences (KP090933) of this strain share $99.5 \%$ homology with Genotype \#1 AB067721, although possible molecular heterogeneity of ITS sequences of this wildtype fungal strain was not reported under the metagenomic fosmid library construction and the observation of multicellular polykaryotic phenomena [9]. The L0106 strain was cultured in a medium favoring $H$. sinensis growth (containing a wide selection of carbon sources) at $16^{\circ} \mathrm{C}$ for 3,6 or 9 days in 200-liter submerged stirred fermenters, followed by reverse transcription of pure mRNA isolated from the mycelia of the L0106 strain. Transcriptome profiling detected a total of 25,511 unigenes from the 3-day culture, 25,214 unigenes from the 6-day culture, and 16,245 from the 9-day culture. These authors also reported in a supplementary file that $71 \%-78 \%$ of the L0106 transcriptome reads could be mapped to the reference genome and that $31-33 \%$ of reads could be mapped to reference genes, indicating the heterokaryosis of the L0106 strain. (Note: the information provided in a different report [9] suggests that the genome survey may have been performed on the L0106 strain, probably through metagenomic fosmid library end-sequencing, and the heterokaryotic results were used as the reference for transcriptomic gene mapping. In addition, Bushley et al. [52] reported the 
observation of two nuclei in hypha using fluorescent staining for mycelia.) All of these transcriptome data identified greater numbers of actively transcribed genes from the natural C. sinensis insect-fungi complex [85] or from the cultures of the wild-type fungal isolate [9] than the total number of genes (both actively expressed and silent) identified from the genome-wide sequencing of a purified anamorphic $H$. sinensis strain [16], thus confirming the coexistence of multiple fungi in natural $C$. sinensis and supporting the notion that the considerable therapeutic functions result from the symbiotic activities of multiple fungal sources and the dead body of the larva of the family Hepialidae. Although Xiang et al. [85] did not specify the maturation status of the specimens tested, and Liu et al. [9] profiled the transcriptome genes in the cultures of a fungal isolate, the significant changes in proteomic polymorphisms [10] indicate dynamic changes in the expression of transcriptomic genes in the different compartments of $C$. sinensis during maturation. All of the proteins that are differentially expressed in the different compartments of $C$. sinensis and altered with maturation, along with the varied profiles of their component chemicals (lipids, polysaccharides, nucleotides and other chemical constituents), contribute to the varied efficacy profiles and potency of the therapeutic activities of natural $C$. sinensis, for the initial discovery of this highly valued TCM product was based on its therapeutic efficacy and unique appearance [12,4,7,10,11,19,86-87].

\section{Molecular systematics for the genetically heterogeneous $C$. sinensis}

Microcosmic molecular systematic studies comparing nrDNA ITS sequences have been used to determine the taxonomic status of the examined specimens. The detection of $H$. sinensis ITS sequences in the genomic DNA of natural $C$. sinensis from 1999-2003 consistently demonstrated the molecular "homogeneity" of the PCR amplicons, providing molecular systematic evidence for the "correct" anamorph and supporting the hypothesis that $H$. sinensis is the sole anamorph of $O$. sinensis [63-69]. The success of these molecular studies of natural C. sinensis provided inertia for the continuing assumptions accepted in later years $[14,28,29,51,65]$, regardless of the documented controversies as to the isolation of $C$. sinensis-associated fungi (22 species spanning 13 genera) [17-18,27] and the subsequent identification of additional fungi [25,44-46,48,51].

Chen et al. [8] first reported the molecular heterogeneity of $C$. sinensis-associated fungi using a PCR amplicon cloning technique. However, insufficient attention was given to these "all-or-none" qualitative research findings, and instead, the disproportionate amplicon clones selected for examining the ITS sequences of multiple fungi were overemphasized, unfortunately leading to an improper conclusion. The observations of molecular heterogeneity have attracted scientific attention since 2005, and there have been reports of the ITS sequences of multiple C. sinensis-associated fungi, including Cladosporium macrocarpum, Geomyces pannorum, Neosetophoma samarorum, Paecilomyces hepiali, Phaeosphaeria pontiformis, Pseudogymnoascus roseus, and Tolypocladium sinense (in total, more than 90 species spanning at least 37 genera), in addition to the multiple $O$. sinensis genotypes described above $[11,25,26,27,42,44-49,51,59$ 60]. Southern blotting, RFLP assay, SSCR assay, SNP MS genotyping, nested-PCR, qPCR, amplicon sequencing and amplicon cloning sequencing have been used to identify several genotypes of $O$. sinensis mutant fungi with multiple, scattered point mutations from natural $C$. sinensis [11-12,25-26,49,51,54-62]. Therefore, the notion that $H$. sinensis is the sole anamorph of natural $C$. sinensis faces substantial challenges based on both mycological and molecular systematic evidence and remains a hypothetical assumption requiring further analysis that 
should be conducted strictly according to Koch's Postulates.

Although aforementioned microcosmic molecular examinations have revealed individual taxonomies through ITS sequencing, individual fungal ITS and other sequences do not represent natural $C$. sinensis due to its genetic heterogeneity. The microcosmic ITS sequence data have fueled speculations, hypotheses and non-conclusive debates, similar to blind men each touching a portion of an elephant. To address this shortcoming, macrocosmic holistic molecular marker polymorphism analysis has been used as a component of overall molecular systematics strategies to profile natural $C$. sinensis as a holistic entity and to compare the holistic polymorphic similarities of the systems without the requirement of precise examinations of the DNA sequences or the individual taxonomies of the component fungi. These macrocosmic molecular techniques include AFLP (Amplified Fragment Length Polymorphism), CAPS (Cleaved Amplified Polymorphic Sequence), DAF (DNA Amplified Fingerprints), ISSR (Inter-Simple Sequence Repeat), RAPD (Random Amplified Polymorphic DNA), RFLP, SCAR (Sequence Characterized Amplified Regions), SSCP (single-strand conformation polymorphism), and SSR (Simple Sequence Repeat) [88-89]. Among these methodologies, RAPD molecular marker polymorphism analysis is the most frequently used technique for comparing overall similarities or dissimilarities (genetic distances) and exploring the phylogenetic cluster relationship between the test systems [14,62,90-97], although it has been suggested that ISSR may be more sensitive than RAPD [81,98-99], and metagenomics approaches may demonstrate advances in qualitative studies of microbial genetic diversity and molecular ecology [100-102]. A few issues regarding holistic molecular marker polymorphism studies are reviewed below.

\subsection{Selection of RAPD random primers}

RAPD and ISSR molecular marker polymorphism analyses compare the migration and density (abundance) of PCR amplicons in non-denaturing agarose gel electrophoresis by computing integral similarity and constructing phylogenetic (cluster) trees [13-14,62,81,90-99]. Prior to the agarose gel electrophoresis, these approaches require the use of a plurality of primers for PCR amplification of the genomic DNA templates isolated from the examined systems. The selection of the type and quantity of random primers, therefore, becomes crucial for the unbiased design of RAPD and ISSR marker polymorphism studies to obtain unbiased profiles of the holistic differences between the tested samples [13,17,50,62,93].

After screening 20-65 random primers, as many as 8-29 primers have been selected for the $C$. sinensis studies based on the number and density of the DNA amplicon moieties in the gel images [90-97]. The use of only a few random primers without reporting the objectivity and representativeness of the selection could lead to bias in the data analysis and thus bias in the study conclusions $[17,50,62,93]$ when $C$. sinensis samples and fungal strain samples were not profiled as a whole, resulting in the inaccuracy of holistic comparisons, interpretations and conclusions [14]. Thus, scientists have warned that the selection of the type and number of random primers is critical for the interpretations and conclusions drawn from RAPD and ISSR results $[17,50,62,93]$.

\subsection{Computational biology algorithms for polymorphism similarity analysis}

Macrocosmic analysis of molecular marker polymorphisms relies upon similarity computation and phylogenetic (cluster) tree construction. Previous studies have consistently used the PCR amplicon densityunweighted algorithm known as the Nei-Li equation [103] for similarity computations. This or similar density-unweighted algorithms 
have also been used in SSCP and ISSR studies $[25,51,81,99]$.

The Nei-Li algorithm (or similar) was designed to analyze "all or none" data, to compare pure systems in pairs and, in particular, to analyze the loss of restriction sites due to mutations [103]. The proper use of this algorithm has 2 prerequisites: (1) all matched DNA pairs in the electrophoretic lanes being compared must have essentially the same densities; and (2) all DNA amplicons must be well separated from the adjacent DNA moieties with similar molecular weights and conformations by electrophoresis [62,93]. Clearly, this algorithm is unsuitable for RAPD or ISSR studies of $C$. sinensis as an insect-fungi complex containing multiple fungi, and a series of new ZUNIX arithmetic methods (www.ebioland.com/ZUNIX.htm; Beijing Bioland Technology, 2013) was developed for density-weighted similarity computation $[62,93]$. The ZUNIX equations arithmetically consider the following: (1) the unmatched DNA (or protein or other chemical) bands and their densities, (2) differences in the density of the matched DNA (or protein, or other chemical) bands (or peaks, or areas under the curves), and (3) the ability to compare multiple samples. The density-weighted ZUNIX equations define similarity as the total density of all common parts present in the matched DNA bands of the samples being compared divided by the total density of all bands across the samples [93]. The ZUNIX equations are mathematically general, with no specific prerequisites, and govern all conditions, including the special cases under the strict prerequisites set forth by the Nei-Li equation [103], and they accurately capture all of the molecular information buried in the amplicon DNA bands (both the density and the migration speed in gel electrophoresis) in the RAPD (or ISSR, SSCP, or similar techniques) gel images, which are partially lost or even significantly lost when incorrectly using the density-unweighted Nei$\mathrm{Li}$ equation [103]. Consequently, misuse of the Nei-Li equation [103] in C. sinensis holistic polymorphism studies when the sample systems do not meet the specific prerequisites may lead to inaccurate calculations of overall similarities and questionable conclusions [81,92]. The mathematically general, density-weighted ZUNIX equations can also accurately calculate the similarities of proteomic polymorphisms of multiple $C$. sinensis samples $[10,93]$.

\subsection{Density-weighted algorithms for phylogenetic (cluster) tree construction}

Phylogenetic analysis in previous RAPD and ISSR studies of $C$. sinensis primarily used PCR amplicon density-unweighted UPGMA (Unweighted Pair Group Method with Arithmetic Mean) algorithms to construct phylogenetic trees $[14,81,90,94,97,99]$. Consistent with the computation of similarity, constructing phylogenetic trees using densityunweighted algorithms in the holistic analysis negates the differences between high- and low-density DNA amplicons or between the complete and incomplete separation of DNA amplicon moieties on agarose gel electrophoresis and their impact on the weights in exploring phylogenetic similarity and dissimilarity, leading to errors in constructing phylogenetic trees. Ni et al. [93] demonstrated the inaccurate construction of a phylogenetic tree improperly using the density-unweighted algorithm, reflecting the inability to capture and analyze all molecular information buried in the DNA bands (both the density and the migration speed in agarose gel electrophoresis) in RAPD gel images, whereas the density-weighted algorithm corrected such analytical errors. Thus, the selection of different clustering algorithms with or without consideration of the densities and incomplete separations of the DNA moieties (or protein, or chemical moieties) greatly impacts the data analysis and study conclusions, and the density-unweighted algorithms are not suitable for studies of $C$. sinensis, which contains multiple intrinsic fungi. 
As indicated by Ni et al. [93], PAUP 4.0B requires semi-quantitative scoring prior to phylogenetic tree construction, which may slightly reduce the sensitivity in handling fully quantitative density data. Therefore, $\mathrm{Ni}$ et al. [93] performed their clustering analysis using software with full quantitative capacity, such as Cluster3.0, JMP9, and SPSS, and they demonstrated that the fully quantitative algorithms placed $H$. sinensis in a separate clade from the main $C$. sinensis cluster at a large rescaled distance. Both the semi- and fully quantitative methods, however, exhibited advantages in capturing all molecular information and accurately constructing phylogenetic trees in the $C$. sinensis molecular and proteomic polymorphism studies $[10,62,93]$. Other advantages of software for fully quantitative clustering include ease of use and accurate quantitation, but the algorithms provided by the software do not include bootstrap value calculation, whereas the semi-quantitative clustering algorithm provided by PAUP 4.0B calculates the bootstrap value (usually Bootstrap=1000). The type(s) of software should be considered when designing RAPD, ISSR, SSCP and other holistic profile comparison studies, although both fully and semi-quantitative clustering algorithms can generally be used for $C$. sinensis molecular and proteomic polymorphism studies and chemical fingerprint studies.

\section{Summary}

This review summarizes the scientific debates in the molecular studies of $O$. sinensis fungi from natural $C$. sinensis specimens, the natural fungal (species) complex of multiple fungi and the dead bodies of larvae of the family Hepialidae. The genetic heterogeneity of natural $C$. sinensis and multiple fungi under the name $O$. sinensis indicate that the IMA Amsterdam Declaration $1 \mathrm{~F} 1 \mathrm{~N}$ can be properly implemented in the $C$. sinensis research field only after careful confirmation of $O$. sinensis as truly "One Fungus" and of the identification of one of the fungi in natural
$C$. sinensis as the true anamorph of $O$. sinensis strictly following Koch's Postulations [28,39-41]. Prior to such scientific confirmation, all 3 hypotheses relating to the $O$. sinensis fungi under Sections 2,1,2.2, and 2.3 should be treated scientifically and equally to avoid academic unfairness. An academic consensus to end this decades-long debate needs to be reached only after such scientific confirmation by a committee of multidisciplinary experts. In addition to the problematic use of the name $O$ sinensis for the multiple homokaryotic anamorphic fungi and the multicellular heterokaryotic teleomorph ascospores, the natural insect-fungi complex should not use the same Latin name of $O$. sinensis to avoid academic confusion if this name is used for the fungi [30]. The multiple fungi that differentially exist in the $C$. sinensis compartments, possibly in the form of bi/trinucleate heterokaryons or fungal (species) complexes or other wild-type symbiotic relationships, undergo asynchronous alterations during $C$. sinensis maturation, resulting in altered integral molecular marker polymorphisms and proteomic polymorphisms that represent a dynamically altered holistic entity of natural $C$. sinensis. The symbiosis of multiple $C$. sinensisassociated fungi in the entire course of the $C$. sinensis lifecycle should be carefully studied to address fundamental questions concerning the mystery of natural $C$. sinensis biology (including the anamorph-teleomorph connection of $O$. sinensis) and the mass production of artificial C. sinensis to supplement this scarce, precious natural resource that suffers from disproportionately high market demand $[13,50]$.

\section{Acknowledgments}

This study was supported by a research grant (2013BAD16B012) awarded to Professor YLL by China Ministry of Science and Technology: Studies on screening of high-quality lines of insect (the family 
Hepialidae) for Cordyceps sinensis and construction of a demonstration base.

The authors are grateful to Profs. Mu Zang, Ruqin Dai, Zengzhi Li, Yinglan Guo, Ping Zhu, Yan Song, Luqun Ni, Xuanwei Zhou, Yuguo Zheng, and Drs. Yanjiao Zhou, Rongfang Wang, Yu Xia, Jiagang Zhao for consultation.

\section{References}

1. Song, L. R; Hong, X.; Ding, X.L.; Zang, Z. Y. Monographs of modern pharmacy of Chinese traditional medicine. People's Health Press, Beijing, 2001; pp733-737.

2. Xu, J.T. Chinese Medicinal Mycology. Beijing Medical University and China Peking Union Medical University Press, Beijing, 1997; pp68-126.

3. Lu, D. Western records and studies of the Chinese caterpillar fungus to the beginning of the 20th century. J Fungal Res, 2014, 12(4), 233-244. DOI: 10.13341/j.jfr.2014.0043

4. Lo, H.C.; Hsieh, C.; Lin, F.Y.; Hsu, T.H. A systematic review of the mysterious caterpillar fungus Ophiocordyceps sinensis in Dong-Chong-Xia-Cao and related bioactive ingredients. $J$ Tradit Compliment Med 2013, 3(1), 16-32. doi: 10.4103/2225-4110.106538

5. Zhou, X.W.; Li, L.J.; Tian, E.W. Advances in research of the artificial cultivation of Ophiocordyceps sinensis in China. Crit Rev Biotechnol, 2014, 34(3), 233-243. DOI: $10.3109 / 07388551.2013 .791245$

6. Zhu, J.-S.; Halpern, G.M.; Jones, K. The scientific rediscovery of an ancient Chinese herbal medicine: Cordyceps sinensis: Part I \& Part II. J Alternative Complimentary Med, 1998, 4(3), 289-303; \& 4(4), 429-457. DOI: $\underline{10.1089 / \mathrm{acm} .1998 .4 .3-289} \&$ DOI: 10.1089/acm.1998.4.429

7. Li, C.L.; Tan, N.Z.; Barger, J.L.; Zhang, Y.; Ferguson, S.B.; Prolla, T.A.; Zhu, J.-S.
Combined use of whole-gene expression profiling technology and mouse lifespan test in anti-aging herbal product study. Proceeding of New TCM Products Innovation and Industrial Development Summit. Zhejiang University Publishing, Hangzhou, 2011; pp443-448. DOI: 10.13140/RG.2.1.5008.5928

8. Chen, Y.Q.; Hu, B.; Xu, F.; Zhang, W.M. Genetic variation of Cordyceps sinensis, a fruit-body-producing entomopathegenic species from different geographical regions in China. FEMS Microbiol Lett, 2004, 230, 153-158. DOI: 10.1016/S03781097(03)00889-9/full

9. Liu, Z.Q.; Lin, S.; Baker, P.J.; Wu, L.F.; Wang, X.R.; Wu, H.; Xu, F.; Wang, H.Y.; Brathwaite, M.E.; Zheng, Y.G. Transcriptome sequencing and analysis of the entomopathogenic fungus Hirsutella sinensis isolated from Ophiocordyceps sinensis. BMC Genomics, 2015, 16, 106123. DOI: $10.1186 / \mathrm{s} 12864-015-1269-\mathrm{y}$

10.Dong, Y.Z.; Zhang, L.J.; Wu, Z.M.; Gao. L.; Yao, Y.S.; Tan, N.Z.; Wu, J.Y.; Ni, L.Q.; Zhu, J.-S. Altered proteomic polymorphism in the caterpillar body and stroma of natural Cordyceps sinensis during maturation. PLoS ONE, 2014, 9(10), e109083. doi: 10.1371/journal.pone.0109083

11.Zhu, J.-S.; Guo, Y.L.; Yao, Y.S.; Zhou, Y.J.; Lu, J.H.; Qi, Y.; Chen, W.; Zheng, T.Y.; Zhang, L.; Wu, Z.M.; Zhang, L.J.; Liu, X.J.; Yin, W.T. Maturation of Cordyceps sinensis associates with coexistence of Hirsutella sinensis and Paecilomyces hepiali DNA and dynamic changes in fungal competitive proliferation predominance and chemical profiles. J Fungal Res, 2007, 5(4), 214224. DOI: 10.3969/j.issn.16723538.2007.04.009

12.Zhu, J.-S.; Zhao, J.G.; Gao, L.; Li, X.H.; Zhao, J.Q.; Lu, J.H. Dynamically altered expressions of at least 6 Ophiocordyceps sinensis mutants in the stroma of Cordyceps sinensis. J Fungal Res, 2012, 
10(2), 100-112. DOI: 10.3969/j.issn.1671167X.2012.03.025

13. Zhu, J.-S.; Wu, J.Y. Genetic heterogeneity of natural Cordyceps sinensis with coexistence of multiple fungi. Chin $J$ Cell Biol, 2015, 37(2), 284-298. DOI: 10.11844/cjcb.2015.02.0284

14. Wei, X.L.; Yin, X.C.; Guo, Y.L.; Shen, N.Y.; Wei, J.C. Analyses of molecular systematics on Cordycepssinensis and its related taxa. Mycosystema, 2006, 25(2), 192-202. http://europepmc.org/abstract/cba/618538

15. Guo, Y.L.; Xiao, P.G.; Wei, J.C. On the biology and sustainable utilization of the Chinese medicine treasure Ophiocordyceps sinensis (Berk.) Sung, G.H. et al. Modern Chin Med, 2010, 12(11), 3-

8.http://en.cnki.com.cn/Article_en/CJFDT OTAL-YJXX201011002.htm

16. Hu, X.; Zhang, Y.J.; Xiao, G.H.; Zheng, P.; Xia, Y.L. Genome survey uncovers the secrets of sex and lifestyle in caterpillar fungus. Chin Sci Bull, 2013, 58, 28462854.DOI: 10.1007/s11434-013-5929-5

17. Jiang, Y.; Yao, Y.J. A review for the debating studies on the anamorph of Cordyceps sinensis. Mycosistema, 2003, 22(1), 161-176.http://www.docin.com/p1515786037.html

18. Liang, Z.Q. Anamorphs of Cordyceps and their determination. Southwest Chin J Agricult Sci, 1991, 4(4), 1-8. http://www.cnki.com.cn/Article/CJFDTot al-XNYX199104000.htm

19. Liang, Z.Q.; Han, Y.F.; Liang, J.D.; Dong, $\mathrm{X}$.; Du, W. Issues of concern in the studies of Ophiocordyceps sinensis. Microbiol Chin, 2010, 37(11), 1692-1697. http://www.docin.com/p-362612272.html

20. Xiao, Y.Y.; Chen, C.; Dong, J.F.; Li, C.R.; Fan, M.Z. Morphological observation of ascospores of Ophiocordyceps sinensis and its anamorph in growth process. $J$ Anhui Agricult Univ, 2011, 38(4), 587-591. DOI: 34-1162/S.20110624.1628.030

21. Yang, J.Y. Identifying methods of Cordyceps conidial forms. J Qinghai
Normal Univ Nat Sci, 2005, 1, 77-80. http://www.docin.com/p-13180624.html

22. Zhang, P. Advances on cordyceps genus fungi research. $J$ Biol, 2003, 20(6), 43-45. Doi: 1008-9632(2003)06-0043-0

23. Schmeisser, C.; Steele, H.; Streit, W. Metagenomics, biotechnology with nonculturable microbes. Appl Microbiol Biotechnol, 2007, 75, 955-962. DOI: 10.1007/s00253-007-0945-5

24. Tjedje, J.M.; Asuming-Brempong, S.; Nüsslein, K.; Marsh, T.L.; Flynn, S.J. Opening the black box of soil microbial diversity. Appl Soil Ecol, 1999, 13, 109122. doi:10.1016/S0929-1393(99)00026-8

25.Zhang, Y.J.; Sun, B.D.; Zhang, S.; Wangmu; Liu, X.Z.; Gong, W.F. Mycobiotal investigation of natural Ophiocordyceps sinensis based on culturedependent investigation. Mycosistema, 2010a, 29(4), 518-527. http://www.docin.com/p-466261366.html

26. Zhu, J.-S.; Gao, L.; Li, X.H.; Yao, Y.S.; Zhao, J.Q.; Zhou, Y.J. Maturational alterations of oppositely orientated rDNA and differential proliferations of CG:ATbiased genotypes of Cordyceps sinensis fungi and Paecilomyces hepiali in natural Cordyceps sinensis. Am J Biomed Sci, 2010 2(3): 217-238. doi: 10.5099/aj140200082

27. Barseghyan, G.S.; Holliday, J.C.; Price, T.C.; Madison, L.M.; Wasser, S.P. Growth and cultural-morphological characteristics of vegetative mycelia of medicinal caterpillar fungus Ophiocordyceps sinensis G.H. Sung et al. (Ascomycetes) isolates from Tibetan Plateau (P.R. China). Intl $J$ Med Mushrooms, 2011, 13(6), 565-581. DOI: 10.1615/IntJMedMushr.v13.i6.90

28. Zhang, S.; Zhang, Y.J.; Shrestha, B.; Xu, J.P.; Wang, C.S.; Liu, X.Z. Ophiocordyceps sinensis and Cordyceps militaris: research advances, issues and perspectives. Mycosystema, 2013a, 32(4), 577-597. Doi: ISSN1672-6472.CN11$\underline{5180 / Q}$ 
29. Zhang, S.; Zhang, Y.J.; Liu, X.Z.; Zhang, $\mathrm{H}$,; Liu, D,S. On the reliability of DNA sequences of Ophiocordyceps sinensis in public data bases. $J$ Ind Microbiol Biotechnol, 2013b, 40, 365-378. DOI: 10.1007/s10295-012-1228-4

30. Yao, Y.S.; Zhu, J.-S. Indiscriminate use of the Latin name for natural Cordyceps sinensis and Ophiocordyceps sinensis fungi. Chin J Chin Mater Med, 2016, 41(7), (in press)

31. Berkeley, M.J. On some Entomogenous Sphaeriae. London J Botany, 1843, 2, $205-$ 211.

http://cordyceps.cgrb.oregonstate.edu/pag es/some-entomogenous-sphaeriae

32. Berkeley, M.J. On some Entomogenous Sphaeriae. $J$ Proceedings LinneanSoc London (Botany), 1857, 1(4), 157-159. http://www.mycobank.org/BioloMICS.asp $\mathrm{x}$ ? TableKey $=14682616000000061 \& \mathrm{Rec}=$ 40892\&Fields $=$ All

33. Pratt, A.E. To the Snows of Tibet through China. Longmans, Green, and Co., London, 1892; pp16-17, 187-188. https://archive.org/details/tosnowsoftibetth 00pratrich

34. Lloyd, C.G. Cordyceps sinensis, from N. Gist Gee, China. Mycol Notes, 1918, 54, 766-780.

35. Sung, G.H.; Hywel-Jones, N.L.; Sung, J.M.1; Luangsa-ard， J.J.; Shrestha, B.; Spatafora, J.W. Phylogenetic classification of Cordyceps and the clavicipitaceous fungi. Stud Mycol, 2007, 57(1), 5-59. doi:10.3114/sim.2007.57.01

36. Ren, Y.; Wan, D.G.; Lu, X.M.; Guo, J.L. The study of scientific name discussion for TCM Cordyceps. LisShenzhen Med Material Med Res, 2013 24(9), 2211-2212. doi:10.3969/j.issn.1008-0805.2013.09.075

37.Zhang, Y.J.; Li, E.W.; Wang, C.S.; Li, Y.L.; Liu, X.Z. Ophiocordyceps sinensis, the flagship fungus of China: terminology, life strategy and ecology. Mycol, 2012, 3(2), 2-10. DOI: 10.1080/21501203.2011.654354

38. Zhao K, Li Y, Zhang H. Role of dongchongxiacae (Cordyceps) in prevention of contrast-induced nephropathy in patients with stable angina pectoris. J Tradit Chin Med, 2013, 33(3), 283-286.

http://www.ncbi.nlm.nih.gov/pubmed/240 24319

39. Hawksworth, D.L.; Crous, P.W.; Redhead, S.A.; Reynolds, D.R.; Samson, R.A. (and 83 other signees). The Amsterdam Declaration on Fungal Nomenclature. IMA Fungus, 2011, 2(1), 105-112. doi: 10.5598/imafungus.2011.02.01.14

40. IMA. Reports: One fungus $=$ which name? IMA Fungus, 2012, 3(1), 10-14. http://www.imafungus.org/Issue/31/05.pdf

41. Taylor, J.W. One fungus = one name: DNA and fungal nomenclature twenty years after PCR. IMA Fungus, 2011, 2(2), 113-120. 10.5598/imafungus.2011.02.02.01

42. Chen, J.P.; Zhang, W.Y.; Lu, T.T.; Li, J.; Zheng, Y.; Kong, L.D. Morphological and genetic characterization of a cultivated Cordyceps sinensis fungus and its polysaccharide component possessing antioxidant property in $\mathrm{H} 22$ tumor-bearing mice. Life Sci, 2006, 78(23), 2742-2748. doi: 10.1016/j.lfs.2005.10.047

43. Dong, C.H.; Guo, S.P.; Wang, W.F.; Liu, X.Z. Cordyceps Industry in China. Mycol: An Intl J Fungal Biol, 2015, 1-9. DOI: 10.1080/21501203.2015.1043967

44. He, S.Q.; Jin, X.L.; Luo, J.C.; Wang, C.M.; Wang, S.R. Morphological characteristics and submerged culture medium screening of a strain of Geomyces pannorum isolated from Cordyceps sinensis. Chin $J$ Grassland, 2010, 32(z1), 70-75. http://www.docin.com/p-213145063.html

45. He, S.Q.; Jin, X.L.; Luo, J.C.; Wang, C.M. Morphological characteristics and submerged culture medium screening of Pseudogymnoascus roseus. Microbiol Chin, 2011, 38(9), 1371-1376. http://www.cnki.com.cn/Article/CJFDTot al-WSWT201109010.htm

46. Jiang, Y.; Yao, Y.J. ITS sequence analysis and ascoma development of 
Pseudogymnoascus roseus. Mycotaxon, 2005, 94, 55-73.

47.Leung, P.H.; Zhang, Q.X.; Wu, J.Y. Mycelium cultivation, chemical composition and antitumour activity of a Tolypocladium sp. fungus isolated from wild Cordyceps sinensis. $J$ Applied Microbiol, 2006, 101(2), 275-283. DOI: 10.1111/j.1365-2672.2006.02930.x

48. Xia, F.; Liu, Y.; Shen, G.L.; Guo, L.X.; Zhou, X.W. Investigation and analysis of microbiological communities in natural Ophiocordyceps sinensis. Can J Microbiol, 2015, 61, 104-111. DOI: 10.1139/cjm2014-0610

49. Yang, J.L.; Xiao, W.; He, H.H.; Zhu, H.X.; Wang, S.F.; Cheng, K.D.; Zhu, P. Molecular phylogenetic analysis of Paecilomyces hepiali and Cordyceps sinensis. Acta Pharmaceutica Sinica, 2008, 43(4), 421-426. DOI: $10.1111 / \mathrm{j} .1745-$ 7254.2008.00754.X

50. Yao, Y.S.; Xie, W.D.; Zhu, J.-S. Genetic heterogeneity of natural Cordyceps sinensis with co-existence of multiple fungi and at least 12 genotype mutants. Proceedings of 2015 The First National Cordyceps Conference and Ophiocordyceps sinensis Industrial Summit. Jinhu City, Jiangsu Province, China. June 27-29, 2015; pp63-82. DOI: 10.13140/RG.2.1.1532.9124

51.Zhang, Y.J.; Zhang, S.; Wang, M.; Bai, F.Y.; Liu, X.Z. High diversity of the fungal community structure in naturallyoccurring Ophiocordyceps sinensis. PLoS $O N E, \quad 2010 b, \quad 5(12)$, e15570. doi: 10.1371/journal.pone.0015570

52. Bushley, K.E.; Li, Y.; Wang, W.J.; Wang, X.L.; Jiao, L.; Spatafora, J.W.; Yao, Y.J. Isolation of the MAT1-1 mating type idiomorph and evidence for selfing in the Chinese medicinal fungus Ophiocordyceps sinensis. Fungal Biol, 2013, 117, 599-610. DOI: 10.1016/j.funbio.2013.06.001

53. Stensrud, Ø.; Schumacher, T.; ShalchianTabrizi, K.; Svegardenib, I.B.; Kauserud, H. Accelerated nrDNA evolution and profound AT bias in the medicinal fungus Cordyceps sinensis. Mycol Res, 2007, 111, 409-415. doi: 10.1016/j.mycres.2007.01.015

54.Xiao, W.; Yang, J.P.; Zhu, P.; Cheng, K.D.; He, H.X.; Zhu, H.X.; Wang, Q. Non-support of species complex hypothesis of Cordyceps sinensis by targeted rDNA-ITS sequence analysis. Mycosystema, 2009, 28(6), 724-730. http://en.cnki.com.cn/Article_en/CJFDTO TAL-JWXT200905024.htm

55.Gao, L.; Li, X.H.; Zhao, J.Q.; Lu, J.H.; Zhu, J.-S. Detection of multiple Ophiocordyceps sinensis mutants in premature stroma of Cordyceps sinensis by MassARRAY SNP MALDI-TOF mass spectrum genotyping. Beijing Da Xиe Xue Bao, 2011, 43(2), 259-266. Doi: 10.3969/i.issn.1671-167X.2011.02.019

56. Gao, L.; Li, X.H.; Zhao, J.Q.; Lu, J.H.; Zhao, J.G.; Zhu, J.-S. Maturation of Cordyceps sinensis associates with alterations of fungal expressions of multiple Ophiocordyceps sinensis mutants with transition and transversion point mutations in stroma of Cordyceps sinensis. Beijing Da Xиe Xие Bao, 2012, 44(3), 454-463. Doi: 10.3969/i.issn.1671167X.2012.03.025

57. Li, Yi.; Jiao, L.; Yao, Y.J. Non-concerted ITS evolution in fungi, as revealed from the important medicinal fungus Ophiocoordyceps sinensis. Molecul Phylogenetics Evolut, 2013, 68, 373-379. DOI: 10.1016/j.ympev.2013.04.010

58. Mao, X.M.; Zhao, S.M.; Cao, L.; Yan, X.; Han, R.C. The morphology observation of Ophiocordyceps sinensis from different origins. J Environmental Entomol, 2013, 35(3), 343-353. DOI: 10.3969/j.issn.16740858.2013.03.11

59. Yao, Y.S.; Zhou, Y.J.; Gao, L.; Lu, J.H.; Wu, Z.M.; Zhu, J.-S. Dynamic alterations of the differential fungal expressions of Ophiocordyceps sinensis and its mutant genotypes in stroma and caterpillar during maturation of natural Cordyceps sinensis. 
J Fungal Res, 2011, 9(1), 37-49, 53. doi: 1672-3538(2011)01-0037-13

60.Zhu, J.-S.; Yao, Y.S.; Gao, L.; Wu, Z.M.; Li, Y.L.; Zhao, J.G. Dynamically altered fungal expressions of Paecilomyces hepiali and 7 Ophiocordyceps sinensis genotypes in natural Cordyceps sinensis. The 10th International Mycological Congress, Bangkok, Thailand, August 7, 2014. Special Symposium 5. DOI: 10.13140/RG.2.1.4013.0964

61. Li, Y.L.; Yao, Y.S.; Ma, S.L.; Zhang, Z.H.; Liu, X.; Xu, H.F.; Wu, Z.M.; Zhu, J.-S. Synergy of fungal complexes isolated from the intestines of Hepialus lagii larvae containing multiple fungi increases infection potency. J Fungal Res, 2016, (in press)

62. Yao, Y.S.; Gao, L.; Li, Y.L.; Ma, S.L.; Wu, Z.M.; Tan, N.Z.; Wu, J.Y.; Ni, L.Q.; Zhu, J.-S. Amplicon density-weighted algorithms analyze dissimilarity and dynamic alterations of RAPD polymorphisms in the integrated microecosystem Cordyceps sinensis. Beijing Da Хие Хие Ваo, 2014, 46(4), 618-628. doi: 10.3969.j.issn.1671-167X.2014.04.027

63. Chen, Y.Q.; Wang, N.; Qu, L.H.; Li, T.H.; Zhang, W.M. Determination of the anamorph of Cordyceps sinensis inferred from the analysis of the ribosomal DNA internal transcribed spacers and $5.8 \mathrm{~S}$ rDNA. Biochem Systemat Ecol, 2001, 29, 597-607. $\quad$ DOI: $10.1016 /$ S03051978(00)00100-9

64. Chen, Y.Q.; Wang, N.; Zhou, H.; Qu, L.H. Differentiation of medicinal Cordyceps species by rDNA ITS sequence analysis. Planta Medica, 2002, 68, 635-639. DOI: $10.1055 / \mathrm{s}-2002-32892$

65. Hao, J.J.; Cheng, A.; Liang, H.H.; Yang, X.L.; Li, A.; Zhou, T.S.; Zhang, W.J.; Chen, J.K. Genetic differentiation and distributing pattern of Cordyceps sinensis in China revealed by rDNA ITS sequences. Chin Tradit Herbal Drugs, 2009, 40(1), 112-116.

http://en.cnki.com.cn/Article_en/CJFDTot al-ZCYO200901037.htm
66. Kinjo, N.; Zang, M. Morphological and phylogenetic studies on Cordyceps sinensis distributed in southwestern China. Mycosci, 2001, 42, 567-574. DOI: $\underline{10.1007 / \mathrm{BF} 02460956}$

67.Liu, Z.Y.; Yao, Y.J.; Liang, Z.Q.; Liu, A.Y.; Pegler, D.N.; Liu, A.Y.; Pegler, D.N.; Chase, M.W. Molecular evidence for the anamorph-teleomorph connection in Cordyceps sinensis. Mycol Res, 2001, 105(7), 827-832. DOI: 10.1017/S095375620100377X

68. Wang, N.; Chen, Y.Q.; Zhang, W.M.; Li, T.H.; Qu, L.H. Molecular evidences indicating multiple origins in the entomogenous Cordyceps. Acta Sci Natural Univ Sunyatseni, 2000, 39(4), 7073.

http://www.cnki.com.cn/Article/CJFDTot al-ZSDZ200004016.htm

69.Zhao, J.; Wang, N.; Chen, Y.Q.; Li, T.H.; $\mathrm{Qu}$, L.H. Molecular identification for the asexual stage of Cordyceps sinensis. Acta Sci Natural Univ Sunyatseni, 1999, 38(1), 122-123. http://www.docin.com/p721692066.html

70.Liu, Z.Y.; Liang, Z.Q.; Liu, A.Y. Investigation on microcycle conidiation of ascospores and conidiogenous structures of anamorph of Cordyceps sinensis. Guizhou Agricultural Sci, 2003, 31(1), 3-5. http://www.docin.com/p-167710092.html

71.Mo, M.H.; Chi, S.Q.; Zhang, K.Q. Microcycle conidiation of Cordyceps sinensis and anamorph isolation. Mycosystema, 2001, 20(4), 482-485. http://www.cnki.com.cn/Article/CJFDTot al-JWXT200104008.htm

72. Lei, W.; Peng, Q.Y.; Zhang, G.R.; Liu, X. The latent infection process of Ophiocordyceps sinensis in host Thitarodes larvae. J Environment Entomol, 2015, 37(2), 387-392. doi: 10.3969/j.issn.1674-0858.2015.02.24

73. Goodhead, I.; Darby, A.C. Taking the pseudo out of pseudogenes. Current Opinion Microbiol, 2015, 23, 102-109. Doi: 10.1016/j.mib.2014.11.012 
74. Roberts, T.C.; Morris, K.V. Not so pseudo anymore: pseudogenes as therapeutic targets. Pharmacogenom, 2013, 14(16), 2023-2034. doi: 10.2217/pgs.13.172

75. Li, W.; Yang, W.; Wang, X.J. Pseudogenes: pseudo or real functional elements? J Genet Genom, 2013, 40(4), 171-177. doi: 10.1016/j.jgg.2013.03.003

76. Muro, E.M.; Ma,h N.; Andrade-Navarro, M.A. Functional evidence of posttranscriptional regulation by pseudogenes. Biochimie, 2011, 93(11): 1916-1921. doi: 10.1016/j.biochi.2011.07.024

77. Pink, R.C.; Carter, D.R. Pseudogenes as regulators of biological function. Essays Biochem. 2013, 54, 103-112. doi: $\underline{10.1042 / \mathrm{bse} 0540103}$

78. Sun, C.; Orozco, O.; Olson, D.L.; Choi, E.; Garber, E.; Tizard, R.; Szak, S.; Sanicola, M.; Carulli, J.P. CRIPTO3, a presumed pseudogene, is expressed in cancer. Biochem Biophys Res Commun, 2008, 377(1), 215-220. doi: $\underline{10.1016 / j . b b r c .2008 .09 .113}$

79. Wen, Y.Z.; Zheng, L.L.; Qu, L.H.; Ayala, F.J.; Lun, Z.R. Pseudogenes are not pseudo any more. RNA Biol, 2012, 9(1), 27-32. doi: 10.4161/rna.9.1.18277

80. Zheng, D.; Gerstein, M.B. The ambiguous boundary between genes and pseudogenes: the dead rise up, or do they? Trends Genet, 2007, 23(5), 219-224. http://www.ncbi.nlm.nih.gov/pubmed/173 82428

81. Xiao, Y.Y.; Cao, Y.P.; Zhang, L.; Li, C.R. Study on genetic diversity of anamorphic isolates from different sources of Ophiocordyceps sinensis by ISSR. J Fungal Res, 2014, 12(3), 154-159.doi: 1672-3538(2014) 03-0154-06

82. Jiao, L. Phylogeographic study on Ophiocordyceps sinensis. Dissertation, Graduate School of Chinese Academy of Sciences, 2010.

83. Jin, Z.X.; Yang, S.H. Progresses and trends of Cordyceps sinensis studies. J Tiajin Med Univ, 2005 11(1): 137-140. doi: 1006-8147(2005)01-0137-04
84.Feng, H.; Jin, Y.S.; Liu, Y.N.; Liu, Z.; Han, Y.N.; Sun, H.B.; Zhang, S.P. Expressed Sequence Tags (EST) from Cordyceps sinensis. World Sci Technol Modern Tradit Chin Med Materia Medica, 2010, 12(4), 604-609. doi: 10.3969/j.issn.1674-3849.2010.04.023

85. Xiang, L.; Li, Y.; Zhu, Y.J.; Luo, H.M.; Li, C.F.; Xu, X.L.; Sun, C.; Song, J.Y.; Shi, L.C.; He, L.; Sun, W.; Chen, S,L. Tanscriptome analysis of the Ophiocordyceps sinensis fruiting body reveals putative genes involved in fruiting body development and cordycepin biosynthesis. Genomics, 2014, 103, 154159. DOI: 10.1016/j.ygeno.2014.01.002

86.Li, S.P.; Song, Z.H.; Dong, T.T.X.; Ji, Z.N.; Lo, C.K.; Zhub, S.Q.; Tsim, K.W.K. Distinction of water soluble constituents between natural and cultured Cordyceps by capillary electrophoresis. Phytomed, 2004, 11, 684-690. doi:10.1016/j.phymed.2003.07.011

87. Wu, Y.S.; Zhou, D.L.; Yan, D.; Ren, Y.S.; Fang, Y.L.; Xiao, X.H.; Du, X.X.; Wang, J. HPLC fingerprint analysis of Cordyceps and mycelium of cultured Cordyceps. Chin J Chin Mater Med, 2008, 33(19), 2212-2214.doi: 100125302(2008)1922212204

88. Bai, Y. DNA molecular marker techniques and their application. J Anhui Agri Sci, 2007, 35(24), 7422-7424. doi: 05176611(2007)24-07422-03

89. Song, D.L.; Zhang, J.; Chen, J.X. DNA molecular markers and their application in Cordyceps sinensis research. Acta Edulis Fungi, 2002, 9(3), 52-55. doi: 10059873(2002)03-52-0

90. Chen, Y.J.; Zhang, Y.P.; Yang, Y.X.; Yang, D.R. Genetic diversity and taxonomic implication of Cordyceps sinensis as revealed by RAPD markers. Biochem Genet, 1999, 37(5), 201-113. doi: 0006-2928/99/0600-0201\$16.00/0

91. Feng, K.; Wang, S.; Hu, D.J.; Yang, F.Q.; Wang, H.X.; Li, S.P. Random amplified polymorphic DNA (RAPD) analysis and the nucleosides assessment of fungal 
strains isolated from natural Cordyceps sinensis. J Pharmaceut Biomed Anal, 2009, 50, 522-526. doi:10.1016/j.jpba.2009.04.029

92. Li, Z.Z.; Huang, B.; Li, C.R.; Fan, M.Z. Molecular evidence for anamorph determination of Cordyceps sinensis (Berk.) Sacc. Mycosystema, 2000, 19(1), 60-64. Doi: 1007-3515(2000)01-00600064

93. Ni, L.Q.; Yao, Y.S.; Gao, L.; Wu, Z.M.; Tan, N.Z.; Zhu, J.-S. Arithmetic methods for similarity computation and cluster tree construction in RAPD analysis on a multifungi microecology system, Cordyceps sinensis. Am J BioMed Sci, 2014, 6(2), 82104. doi: 10.5099/aj140200082

94. Qian, M.; Zeng, W.; Zhang, D.L. RAPD polymorphism analysis on Cordyceps sinensis. LisShenzhen Med Materia Medica Res, 2011, 22(7), 1738-1739. doi: 10.3969/j.issn.1008-0805.2011.07.090

95. Xie, F.; Zhu, Z.X.; Chen, J.J. RAPD and analysis of ITS sequences of Ophiocordyceps sinensis in Gansu. Chin J Microecol, 2011, 23(3), 219-222, 229. Doi: 1005-376X( 2011) 03-0219-04

96. Yu, C.; He, J.L.; Zeng, W.; Deng, B. Fingerprint comparison on Cordyceps sinensis and artificial ones by RAPD. Chin Tradit Herbal Drugs, 2005, 36(2), 274-277. doi: 0253-2670(2005)02-027404

97.Zhang, Y.W.; Chen, Y.J.; Shen, F.R.; Yang, Y.X.; Yang, D.R.; Zhang, Y.P. Study of genetic divergence in Cordyceps sinensis and $C$. crassispors from Northwest of Yunnan by using RAPD. Mycosystema, 1999, 18(2), 176-183. doi: $\underline{1007-3515(1999) 02-0176-0183}$
98.Liang, H.H.; Cheng, Z.; Yang, X.L.; Li, S.; Zhou, T.S.; Zhang, W.J.; Chen, J.K. Genetic variation and affinity of Cordyceps sinensis in Qinghai Province based on analysis of morphologic characters and inter-simple sequence repeat markers. Chin Tradit Herbal Drugs, 2005, 36(12), 1859-1864. doi: 02532670(2005)12-1859-06

99.Zhu, Z.X.; Xie, F.; Zhang, N. Genetic diversity of Ophiocordyceps sinensis in Gansu Province based on inter-simple sequence repeat (ISSR) analyses. Mycosystema, 2011, 30(3), 501-507. doi: ISSN1672-6472 CN11-5180Q

100. Behzad, H.; Gojobori, T.; Mineta, K. Challenges and opportunities of airborne metagenomics. Genome Biol Evol, 2015, 7(5), 1216-1226. doi: 10.1093/gbe/evv064

101. Sharon, I.; Banfield, J.F. Microbiology. Genomes from metagenomics. Sci, 2013, 342(6162), 1057-1058. 10.1126/science. 1247023

102. Ye, D.D.; Fan, M.M.; Guan, Q.; Chen, H.J.; Ma, Z.S. A review on the bioinformatics pipelines for metagenomic research. Zoological Res, 2013, 33(6), 574-585. 10.3724/SP.J.1141.2012.06574

103. Nei, M.; Li, W.H. Mathematical model for studying genetical variation in terms of restriction endonucleases. Proc Natl Acad Sci USA, 1979, 74(10), 52695273.

http://www.ncbi.nlm.nih.gov/pmc/articles/ PMC413122/ 